3-26-2009

\title{
A Statistical Approach to The Life Cycle Analysis of Cumulus Clouds Selected in A Virtual Reality Environment
}

Thijs Heus

Cleveland State University, t.heus@csuohio.edu

Harmen J.J. Jonker

Delft University of Technology, h.j.j.jonker@tudelft.nl

Harry E.A. Van den Akker

Delft University of Technology

Eric J. Griffith

Delft University of Technology

Michal Koutek

Delft University of Technology

Follow this and additional works at: https://engagedscholarship.csuohio.edu/sciphysics_facpub

epraxtofalar fortydeditionalnauthors

How does access to this work benefit you? Let us know!

Publisher's Statement

Copyright 2011 by the American Geophysical Union

\section{Repository Citation}

Heus, Thijs; Jonker, Harmen J.J.; Van den Akker, Harry E.A.; Griffith, Eric J.; Koutek, Michal; and Post, Frits H., "A Statistical Approach to The Life Cycle Analysis of Cumulus Clouds Selected in A Virtual Reality Environment" (2009). Physics Faculty Publications. 228.

https://engagedscholarship.csuohio.edu/sciphysics_facpub/228

This Article is brought to you for free and open access by the Physics Department at EngagedScholarship@CSU. It has been accepted for inclusion in Physics Faculty Publications by an authorized administrator of

EngagedScholarship@CSU. For more information, please contact library.es@csuohio.edu. 


\section{Authors}

Thijs Heus, Harmen J.J. Jonker, Harry E.A. Van den Akker, Eric J. Griffith, Michal Koutek, and Frits H. Post 


\title{
A statistical approach to the life cycle analysis of cumulus clouds selected in a virtual reality environment
}

\author{
Thijs Heus, ${ }^{1,2}$ Harm J. J. Jonker, ${ }^{1}$ Harry E. A. Van den Akker, ${ }^{1}$ Eric J. Griffith, ${ }^{3}$ \\ Michal Koutek, ${ }^{2,3}$ and Frits H. Post ${ }^{3}$ \\ Received 31 July 2008; revised 30 December 2008; accepted 23 January 2009; published 26 March 2009.
}

[1] In this study, a new method is developed to investigate the entire life cycle of shallow cumuli in large eddy simulations. Although trained observers have no problem in distinguishing the different life stages of a cloud, this process proves difficult to automate, because cloud-splitting and cloud-merging events complicate the distinction between a single system divided in several cloudy parts and two independent systems that collided. Because the human perception is well equipped to capture and to make sense of these time-dependent three-dimensional features, a combination of automated constraints and human inspection in a three-dimensional virtual reality environment is used to select clouds that are exemplary in their behavior throughout their entire life span. Three specific cases (ARM, BOMEX, and BOMEX without large-scale forcings) are analyzed in this way, and the considerable number of selected clouds warrants reliable statistics of cloud properties conditioned on the phase in their life cycle. The most dominant feature in this statistical life cycle analysis is the pulsating growth that is present throughout the entire lifetime of the cloud, independent of the case and of the large-scale forcings. The pulses are a self-sustained phenomenon, driven by a balance between buoyancy and horizontal convergence of dry air. The convective inhibition just above the cloud base plays a crucial role as a barrier for the cloud to overcome in its infancy stage, and as a buffer region later on, ensuring a steady supply of buoyancy into the cloud.

Citation: Heus, T., H. J. J. Jonker, H. E. A. Van den Akker, E. J. Griffith, M. Koutek, and F. H. Post (2009), A statistical approach to the life cycle analysis of cumulus clouds selected in a virtual reality environment, J. Geophys. Res., 114, D06208, doi:10.1029/2008JD010917.

\section{Introduction}

[2] The turbulent behavior and transient nature of cumulus clouds make them a challenging topic of study. Due to heavy in-cloud turbulence, a small but systematic signal can easily be hidden among large random fluctuations. Furthermore, when averaged over the entire lifetime of a cloud, a steady state conceptual model may describe a cumulus cloud quite well, but this does not hold for a single instance. Traditionally, a cloud lifetime is split into three phases: (1) a young cloud with a strong vertical growth; (2) a mature cloud, where the inflow of air from the subcloud thermal is assumed to be in balance with detrainment from the cloud into the environment; and (3) a decaying cloud where the underlying thermal has died out and the cloud is slowly mixed away into the environment.

\footnotetext{
${ }^{1}$ Department of Multi-Scale Physics, Delft University of Technology, Delft, Netherlands.

${ }^{2}$ Now at Royal Netherlands Meteorological Institute, De Bilt, Netherlands.

${ }^{3}$ Department of Mediamatics, Delft University of Technology, Delft, Netherlands.

Copyright 2009 by the American Geophysical Union. 0148-0227/09/2008JD010917
}

[3] For as long as cumulus clouds have been studied, there have been studies into the difference between the stages of the lifecycle of clouds. For instance, Malkus [1952] and Scorer and Ludlam [1953] gained a lot of qualitative insight by looking at sequences of photographs taken from cumulus congestus clouds. In these photographs, a pulsating (or bubbly) growth of the clouds is visible. If a mean shear is present, subsequent pulses can grow on the upshear side of the old pulse in clear air. According to Perry and Hobbs [1996] this air might already be more humid. Such a sheared environment facilitates the observation of the pulsating growth. Whether the shear influences the pulsating growth itself, is less clear.

[4] The pulses are often thought to be important for cloud evolution, because each pulse increases humidity and cools the air, thus creating more favorable circumstances for subsequent pulses. However, pulses are also often observed in a system of shallow cumulus clouds. Grinnell et al. [1996] for instance, followed a single, isolated cumulus near Hawaii throughout its lifecycle using radar. French et al. [1999] also followed a single cloud in time, not only with a ground-based radar, but also with airplane measurements, including an airborne cloud radar. Blyth et al. [2005] combined radar with simultaneous penetrations of clouds by multiple air planes. All these studies showed that the 


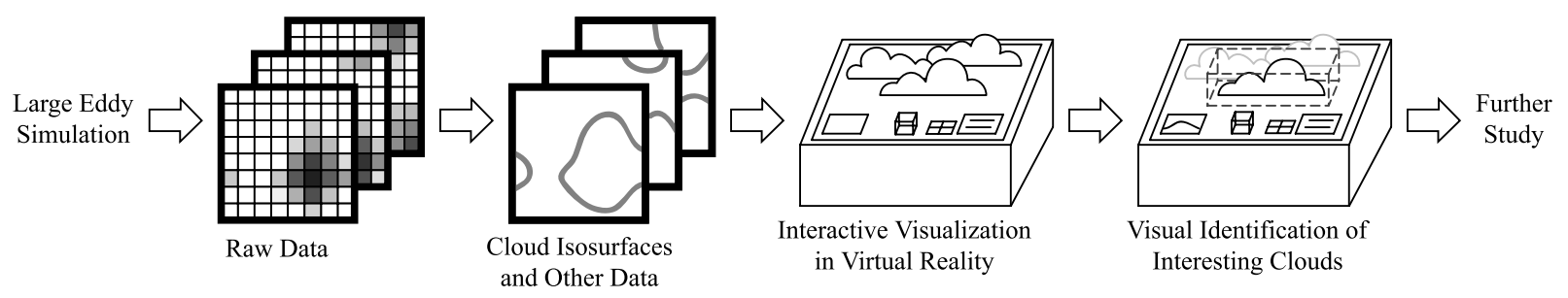

Figure 1. Overview of the workflow of post processing. From Griffith et al. [2005].

conceptual picture of a pulse is useful in describing the evolution of a cumulus cloud in time. Most of the active clouds consist of a sequence of multiple pulses; only the shortest-lived clouds consist of only one pulse, in which case the description of the cloud in terms of pulses reduces to a thermal-based description.

[5] The notion that a cumulus cloud consists of a sequence of pulses does not necessarily mean that each of these pulses travel through the same location as their predecessors. Due to advection of the cloud by the mean wind, subsequent pulses were often observed to emerge in fresh air on the upshear side of the existing cloud [see also Heymsfield et al., 1978; Perry and Hobbs, 1996]. With state-of-the-art three-dimensional radar scanning radar techniques, field campaigns like Rain in Cumulus over the Ocean (RICO [Rauber et al., 2007]) or even in longer time series as currently planned in the Atmospheric Radiation Measurement program (ARM), the life cycle can be studied in ever increasing detail.

[6] Observational studies aside, large eddy simulations (LES) serve as a convenient way to study the behavior of cumulus clouds. With help of LES, Khairoutdinov and Randall [2002] and Kuang and Bretherton [2006] showed that the sequence of various shallow cumulus are important for the transition to deep convection. Although the timescale of this transition, ranging from a few hours to several days, is completely different than the timescale of pulsating growth within a single cloud, the noncontinuous nature is similar. In LES, the three-dimensional, time-dependent fields of all variables are available. However, these complete data sets can easily cause a huge amount of data, which make it difficult to retrieve any needle at all from the haystack.

[7] Ideally, one would implement an automated criterion to isolate in time and space the clouds one wants to study. However, determination of such a criterion is not as straightforward as it may seem. Not only does a representative cloud need to be somewhat isolated from the rest of the ensemble to remove unexpected cloud-cloud interaction from the statistics; but also the cloud needs to be large enough, active enough and possess a certain longevity while still being similar to the many smaller clouds in the ensemble. One way to remedy this problem is to follow a cloud through its life cycle by perturbing the subcloud layer [see, e.g., Bretherton and Smolarkiewicz, 1989; Grabowski and Clark, 1991, 1993a, 1993b; Carpenter et al., 1998; Blyth et al., 2005]. This creates a cloud at a predictable space and time, but the artificially created subcloud thermal may cause anomalous inflow into the cloud. In the spirit of Malkus [1952], Scorer and Ludlam [1953], and French et al. [1999], Zhao and Austin [2005a, 2005b, hereafter ZAA and $\mathrm{ZAB}$ ] chose to hand-pick six clouds from their LES by watching an animation of the cloud field evolving in time. Thanks to their manual selection of clouds, ZAA and ZAB circumvented for a large part the necessity of setting a numerical criterion for an automated selection of representative clouds. Yet the labor-intensive selection process of clouds makes it nontrivial to study more than a few clouds. Therefore a statistically reliable representation of the cloud life cycle that yields quantitative results remains difficult to achieve.

[8] This study aims to move away from looking at the phenomenology of individual clouds, and investigate the lifecycle of enough different cumulus clouds to achieve reliable statistical sampling. In this way, the differences between younger and older clouds, and especially the role of pulses in the evolution of clouds, is studied extensively.

[9] As argued above, the main obstacle to overcome in selecting a large number of clouds is devising a selection process that is sufficiently fast to select a large number of suitable clouds. Instead of aiming at building a highly intelligent automated cloud selection, we make use of the fact that the human eye is well capable of selecting clouds in space and time. In this study, we therefore use a Virtual Reality Environment (VE) to visualize the cloud field. The VE creates a three-dimensional perception of the cloud field, and the VE is optimized to browse through the data set in time. Therefore such an environment is much better equipped than a two-dimensional computer screen to visualize time-dependent three-dimensional fields, and, thus it connects well with both the human perception as well as the physics of the clouds. The observer can, aided by the VE, swiftly select many clouds that fit the requirements. After this selection procedure, further post processing away from the VE can then result in a statistical approach to the life cycle analysis of cumulus clouds.

[10] The remainder of this paper consists of the following parts. In section 2, the methodology used is described, including details of the LES, case descriptions, and the post processing procedure with the VE. As a first look at the results, in section 3 , the life cycles of two individual clouds are treated. The core of this paper, i.e. the statical treatment of the life cycle, is then discussed in section 4, including the (thermo-) dynamical properties of a suitably averaged representation of the life cycle of the entire ensemble of selected clouds. Evidence is given for the mechanism driving the pulsating growth in section 5 .

\section{Methodology}

[11] The workflow of this study is illustrated in Figure 1. First, cloud field data sets are created with large eddy 
simulations, and then the cloud fields are visualized in the VE, where representative clouds are selected. After obtaining the location of these clouds, their properties are further studied outside of the VE.

\subsection{Large Eddy Simulations}

[12] A parallelized version of the Dutch Atmospheric LES (DALES) model, as described by Cuijpers and Duynkerke [1993], was used to run the simulations. With this model, we performed six simulations of the well-documented Barbados Oceanographic and Meteorological EXperiment (BOMEX [Siebesma et al., 2003]), one simulation of BOMEX without large-scale forcings, and one simulation based on observations at the Southern Great Plains (SGP) site of the Atmospheric Radiation Measurement (ARM) program, following the intercomparison by Brown et al. [2002]. The relevant details of the three cases are briefly summarized in this section.

[13] Following Siebesma et al. [2003], the sensible and latent surface heat fluxes in the standard BOMEX case amount to $8 \mathrm{~W} \mathrm{~m}^{-2}$ and $150 \mathrm{~W} \mathrm{~m}^{-2}$, respectively (resulting in a Bowen ratio of $r_{B}=0.05$ ). Lifting condensation level is located around $z_{\mathrm{LCL}}=450 \mathrm{~m}$ and the inversion layer lies between $1500 \mathrm{~m}$ and $2000 \mathrm{~m}$. BOMEX shows a relatively large mean vertical shear, up to $1.8 \mathrm{~m} \mathrm{~s}^{-1} \mathrm{~km}^{-1}$.

[14] Simulations were carried out on a domain of $6.4 \mathrm{~km} \times$ $6.4 \mathrm{~km} \times 3.2 \mathrm{~km}$, with a resolution of $\Delta x=\Delta y=25 \mathrm{~m}$, $\Delta z=20 \mathrm{~m}$. For each of the 6 runs (statistically identical, but each with a different random perturbation of the initial field), 7 hours were simulated, of which the first 3 hours were discarded as spin-up. The complete three-dimensional fields of the 3 components of the velocity $u, v$, and $w$, as well as liquid water potential temperature $\theta_{1}$, the total water content $q_{t}$ and liquid water content $q_{1}$ were recorded every $6 \mathrm{~s}$ of simulation time in short ( 2 bytes) form. This resulted in a $1.7 \mathrm{~TB}$ sized data set, that contains a complete description of the flow.

[15] To gain a better understanding of the clouds, several hypotheses will need to be tested on cases different from the standard BOMEX case. In this study, two additional experiments are done to meet this requirement. Firstly, BOMEX is performed without any large-scale forcings but with otherwise identical specifications. This means that, in comparison with the specifications of Siebesma et al. [2003], (1) no Coriolis force is applied, (2) the geostrophic wind is set to 0 , and (3) no large-scale subsidence, longwave radiative cooling or mean moisture tendency is present. Especially the removal of large-scale subsidence in this simulation results in a significant growth of the atmospheric boundary layer. This means that, unlike the standard BOMEX case, the simulation is not in quasi-steady state anymore, which is reflected in the cloud base reaching $800 \mathrm{~m}$ and an inversion height of $2400 \mathrm{~m}$ during the simulation, although the inversion height remains well below domain height during the $7 \mathrm{~h}$ of simulation. One of the aims of this simulations is to study how the cloud life cycle behaves in an environment without mean shear.

[16] The characteristics of the ARM case include a diurnal cycle with the sensible and latent heat flux peaking at $140 \mathrm{~W} \mathrm{~m}^{-2}$ and $500 \mathrm{~W} \mathrm{~m}^{-2}$, respectively. These surface fluxes are much stronger than for BOMEX, and also result in a much higher Bowen ratio $\left(r_{B}=0.28\right)$, although the Bowen ratio is still relatively low for a continental ABL [Zhu and Zhao, 2008]. While the mean wind is comparable with $\operatorname{BOMEX}\left(U \approx 10 \mathrm{~m} \mathrm{~s}^{-1}\right)$, less vertical shear is observed $\left(0.3 \mathrm{~m} \mathrm{~s}^{-1} \mathrm{~km}^{-1}\right)$ in the bulk of the domain. Similar to the work of Brown et al. [2002], the simulation is performed on a $6.4 \mathrm{~km} \times 6.4 \mathrm{~km} \times 4.4 \mathrm{~km}$ domain with a resolution of $\Delta x=\Delta y=25 \mathrm{~m}, \Delta z=20 \mathrm{~m}$. The first $7 \mathrm{~h}$ are discarded, allowing the cloud layer to fully develop. Clouds are selected from the 8 th to the 13th hour. Typical values for the cloud base height and inversion height are $1100 \mathrm{~m}$ and $2200 \mathrm{~m}$, respectively. Again, all data is written to disk every $6 \mathrm{~s}$.

\subsection{Virtual Reality Environment}

[17] The Virtual Reality Laboratory at Delft University of Technology is the result of a collaboration between the faculty of Computer Science and the faculty of Applied Sciences. One of the goals of this collaboration is to develop new data visualization techniques, which can be directly applied to data from research on physical processes, such as atmospheric phenomena. The virtual environment used in this paper is a product of this collaboration, and it was especially developed for visualizing cloud data [see Griffith et al., 2005].

[18] The virtual environment runs on a Virtual Workbench system. Such a system supports the lab bench metaphor, where users look down on their data rather than being immersed in it. See Figure 2.

[19] Our workbench has a display area that is $179 \times$ $110 \mathrm{~cm}$ in size and has a resolution of $1400 \times 850$ pixels. The system is driven by a dual Pentium 4 Xeon $3.6 \mathrm{GHz}$ computer with 2 gigabytes of RAM. The data to be visualized is stored locally on a RAID, which has read speeds of up to 160 megabytes $\mathrm{s}^{-1}$. Our software is based on OpenSceneGraph (www.openscenegraph.org), which is an open source scene graph library.

[20] The stereo, or "three-dimensional," effect of the virtual reality system is created by showing different, specially rendered images to the user's left and right eyes. In our workbench, this is achieved with the help of two projectors, one for each eye, that are equipped with special filters. We use INFITEC filters (www.infitec.net), which alternatively filter out the left or the right half of the red, blue and green portions of the color spectrum. The projectors are housed inside the workbench and project, via a mirror, the separate images onto the back of the screen. The stereo effect is completed by having users wear special INFITEC goggles that have corresponding filters to block out the image for the other eye.

[21] In our setup, users directly interact with the data by looking at it and with two input devices: a stylus (e.g., for pointing and selection) and a Plexiglas panel (e.g., for slicing through the data). The three-dimensional position and orientation of these devices and the user's head are tracked by a Polhemus Liberty electromagnetic tracker. Our software uses the Virtual Reality Peripheral Network (VRPN [Taylor et al., 2001]) to interface with the tracker. Tracking the user's head position allows us to render the virtual scene from his or her perspective, which then always gives the user a correct view of the scene and enhances the three-dimensional effect. 


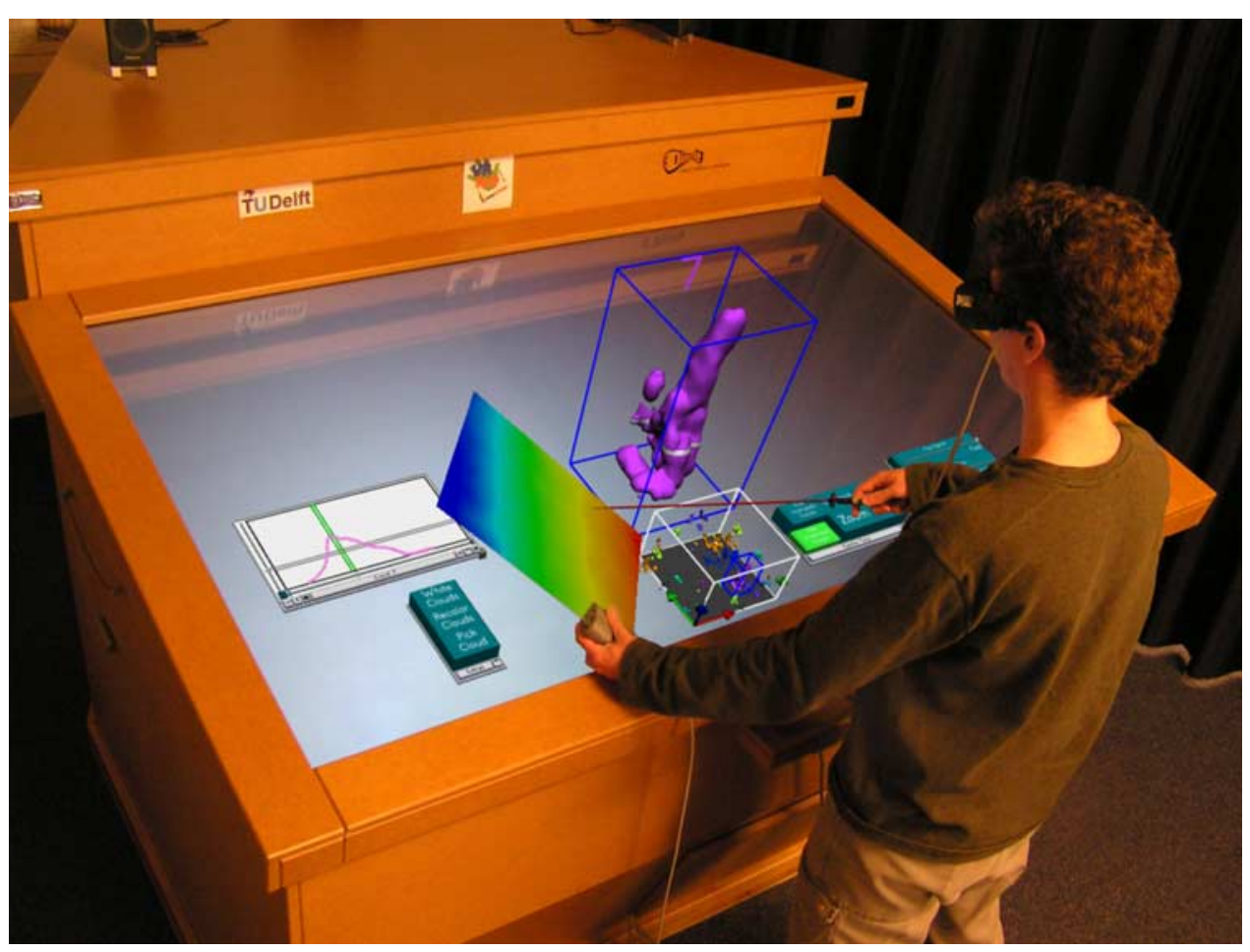

Figure 2. A user working on the virtual environment running on our Virtual Workbench. The user looks down on the data and can use the Plexiglas panel or the stylus to interact with the virtual environment. By wearing electromagnetically tracked INFITEC goggles, the user is provided with a stereo view of the scene, which is rendered from the user's perspective. The cube containing the cloud field can be rotated, zoomed in upon, and browsed through in time. By selecting individual clouds, more information becomes available (e.g., the volume as a function of time).

\subsection{Cloud Selection in Virtual Environment}

[22] In the VE, clouds are visualized by depicting the interface where the liquid water content $q_{1}$ becomes larger than zero. To visualize the clouds, all cloudy grid points that are neighbors (taking the horizontal periodic boundary conditions into account) in four-dimensional space-time are considered to be of the same cloud system and are labeled that way. Additional information on selected clouds, like the evolution of mass or liquid water content in time, is shown in two-dimensional graphs beside the cloud field. Cloud systems that collide or split, systems that already exist at the beginning or still exist at the end of the simulation can be automatically removed from the selection. Additional controls enable the observer to zoom in on a specific region of the cloud field, to rotate the field, or to browse back and forth in time.

[23] Using the VE to browse through the data, active cumulus clouds are selected that, during the bulk of their lifetime, consist of a clear main body with possibly a few chunks broken off. This criterion works rather well during the visible inspection. If a group of clouds is labeled by the VE as one single cloud system, but the observer perceives them as separate clouds that collide at a certain time, the group can be easily dismissed. Like in most observational (airborne) campaigns, the focus of cloud selection lies on active clouds with reasonable life spans. Although this criterion excludes many forced, small and short-lived clouds from the ensemble (as defined for instance by Stull [1985]), the selected clouds aim to be representative of all the active clouds in the cloud field. Using this method for all data sets, 35 clouds are selected from the regular BOMEX case simulations. In total, 3312 clouds are present in the regular BOMEX simulation, including the small, forced clouds and not the aim of this study. From the BOMEX case without large-scale forcings, 12 clouds are selected. From the ARM case, 32 clouds are selected.

\subsection{Validation}

[24] To validate our selection method, the slab averaged profiles averaged over the selected clouds of the vertical velocity $w$, the virtual potential temperature excess $\theta_{\mathrm{v}}^{\prime}$, the liquid water content $q_{1}$, and the liquid water potential temperature $\theta_{1}$ are presented in Figure 3 for the standard BOMEX case. In the same diagrams, the corresponding slab means conditioned over all the clouds in the simulation are shown. The sampling over the selected clouds only matches well with the sampling over the entire cloud field. The selected clouds differ from the entire cloud ensemble only near the top of the cloud layer (above $1500 \mathrm{~m}$ ). There, the cloud statistics are governed by very few (one) large clouds. Usually, those largest clouds are subject to merging and splitting events, and are thus not included in our selection. However, for the bulk of the cloud layer, we can safely assume that the visual cloud selection procedure as described in the previous section yields a set of clouds that is representative for all the (active) clouds in the ensemble. Given the sizable number of selected clouds, and the 

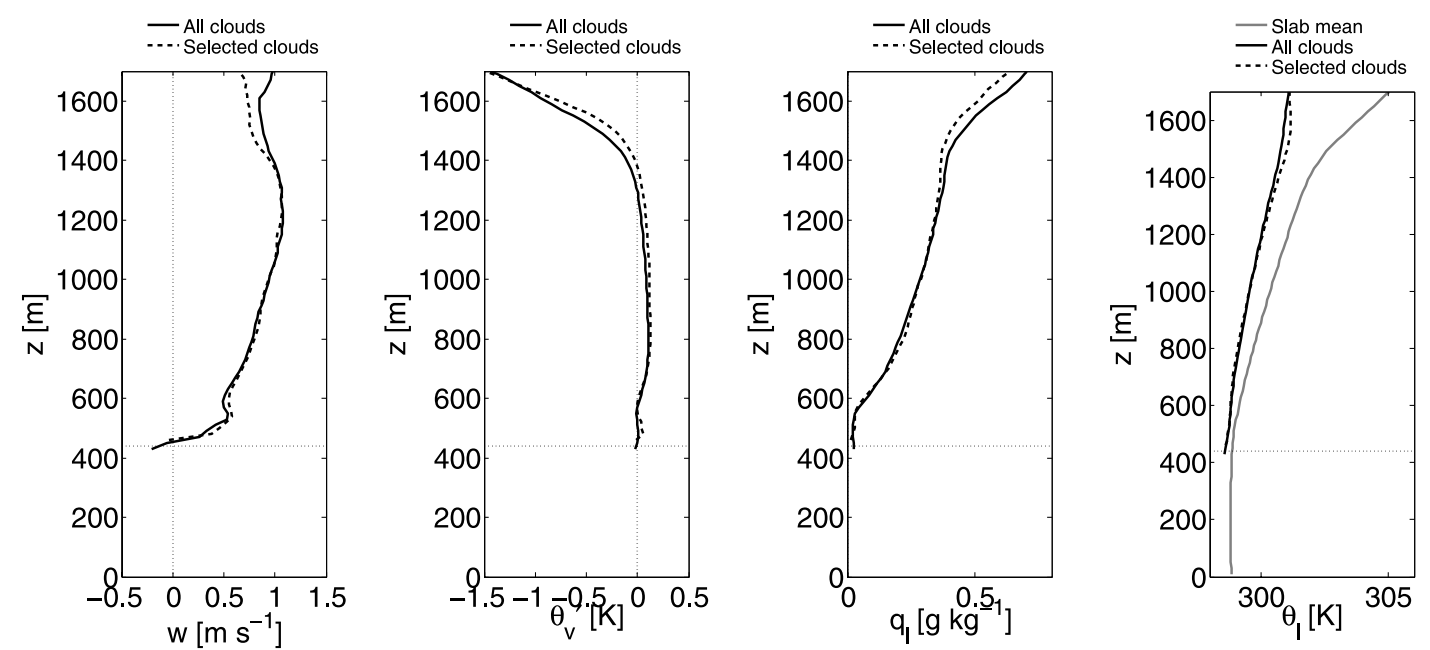

Figure 3. Slab averaged properties sampled over (gray line) the entire domain, (black solid line) all clouds in the simulation, and (dashed line) the selected clouds of the standard BOMEX simulation. From left to right and top to bottom, the vertical velocity $w$, the virtual potential temperature excess $\theta_{\mathrm{v}}^{\prime}$, the liquid water content $q_{1}$, and the liquid water potential temperature $\theta_{1}$.

multiple cases that are studied, a reliable statistical approach of the life cycle of cumulus clouds can be achieved.

\section{Inspection of Individual Clouds}

[25] Before advancing toward a statistical approach, we start with a description of two clouds from the standard run, referred to as cloud A and cloud B, that are exemplary for the entire BOMEX ensemble. To get a good feel for the lifecycle of these two clouds, we look at the evolution in time and height of various slab averaged properties, averaged over the specified cloud:

$$
\bar{\phi}(z, t)=\frac{1}{A_{c}} \int_{A_{c}} \phi d x d y,
$$

with $A_{c}$ the area of the cloud as a function of height and time, and $\phi \in\left\{\rho \Delta z, w, q_{\mathrm{t}}^{\prime}, q_{1}, \theta_{1}^{\prime}, \theta_{\mathrm{v}}^{\prime}\right\}$. If $\phi=\rho \Delta z$, this can be seen as the mass of a slice of the cloud with height $\Delta z$, and density $\rho$. The other variables are the vertical velocity $w$, the total water excess relative to the domain average $q_{\mathrm{t}}^{\prime}$, the liquid water content $q_{1}$, the liquid water potential temperature excess $\theta_{1}^{\prime}$, and the virtual potential temperature excess $\theta_{\mathrm{v}}^{\prime}$. The time-height variations for these six variables are shown for cloud A and cloud B in Figures 4 and 5, respectively. The overlaying vector field in each diagram depicts the in-cloud mean vertical velocity; in other words, it shows the path of a Lagrangian air parcel that is advected through the cloud with the cloud slab averaged velocity. These paths correlate well with the coherent structures in the respective diagrams.

[26] For all 12 diagrams, the presence of the pulses immediately strikes the eye. To assess the typical time between these pulses, we define the pulse interval time $t_{\mathrm{p}}$ as the average time between two minima in the liquid water content at $800 \mathrm{~m}$. Averaged over all 35 clouds, $t_{\mathrm{p}}$ is equal to $408 \mathrm{~s}$; about $50 \%$ of the pulses had a duration between $330 \mathrm{~s}$ and $430 \mathrm{~s}$. This does not match with the convective timescale of the subcloud layer convection [e.g., Stull, 1988]:

$$
t^{*}=\frac{z_{\mathrm{cb}}}{\left(\frac{g}{\Theta_{0}} \overline{w^{\prime} \theta_{v}^{\prime}} z_{\mathrm{cb}}\right)^{\frac{1}{3}}} \approx 720 \mathrm{~s}
$$

with $z_{\mathrm{cb}}=450 \mathrm{~m}$ is taken as the depth of the subcloud layer, $\Theta_{0}$ a reference temperature, $g$ the gravitational acceleration and the surface buoyancy flux $\overline{w^{\prime} \theta_{v}^{\prime}} \approx 1.7 \cdot 10^{-2} \mathrm{~km} \mathrm{~s}^{-1}$. Alternatively, one could look at the active timescale, that scales with the average cloud top height $z_{\text {top }}$ instead of the height of the subcloud layer [Berg and Stull, 2005]: $t_{\text {active }}=$ $t^{*} \frac{z_{\text {top }}}{z_{\mathrm{cb}}} \approx 2100 \mathrm{~s}$. This gives a good estimate of the lifetime of the cloud, but does not match with $t_{\mathrm{p}}$. However, $t_{\mathrm{p}}$ does match reasonably well with the decay time of in-cloud total water content $\tau_{d}$, as derived by Albrecht [1981] and Haiden [1996]:

$$
\tau_{d}=t^{*} \ln \left[1+\left(1+\frac{L}{c_{p}} \frac{\partial q_{s}}{\partial T}\right) \frac{q_{l, 0}}{q_{s}-q_{t}^{e}}\right] \approx 380 \mathrm{~s}
$$

where $L$ is the latent heat of evaporation, $c_{p}$ is the specific heat of air, $q_{s}$ is the saturation specific humidity, $T$ is the temperature, $q_{1,0}$ is the initial liquid water content of the cloud, and $q_{t}^{e}$ is the average ambient total water content. Under the assumption that the similarity between $t_{\mathrm{p}}$ and $\tau_{d}$ is not a coincidence, it appears that the pulsating growth is not just governed by subcloud layer processes, but that the mixing in the cloud layer is an important contributor to the pulsating growth.

[27] This pulse $t_{\mathrm{p}}$ is universal over all clouds in the ensemble; the difference between short-lived clouds and long-lived clouds lies mainly in the number of pulses, as was also observed by ZAA. However, the differences in cloud height between cloud A and cloud B also show that the pulsating growth does not necessarily give a distinction between smaller and larger clouds. If we look at each pulse 

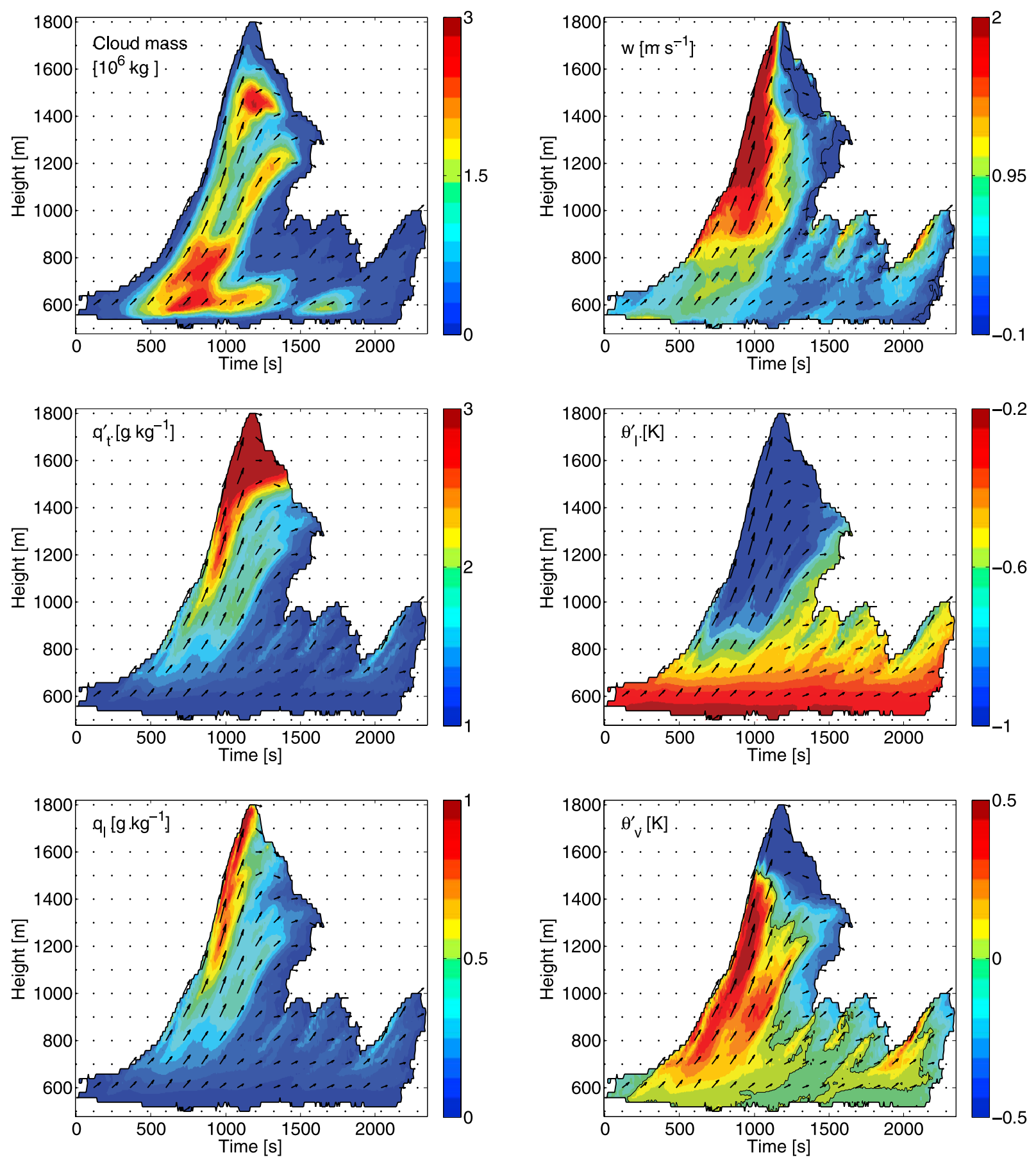

Figure 4. Height-time plots of the cloud area averaged mean properties for cloud A. The thick line denotes the cloud top and cloud base; the thin contour line denotes the 0 -isoline. The vector field denotes the cloud averaged vertical velocity. The diagrams show, from left to right and from top to bottom, the cloud mass, cloud averaged vertical velocity $w$, the cloud averaged excess in total water content $q_{\mathrm{t}}^{\prime}$, the cloud averaged excess in liquid water potential temperature $\theta_{1}^{\prime}$, the cloud averaged liquid water content $q_{1}$, and the cloud averaged excess in virtual potential temperature $\theta_{\mathrm{v}}^{\prime}$.

individually, the emergent view is reminiscent of the shedding thermal of Blyth [1993]. From a nearly neutral cloud base, a short but strongly buoyant thermal head develops, dragging along the region below; this is expressed in the broader and less pronounced pulses in the average vertical velocity. Near the top of the pulse, the cloud becomes negatively buoyant first, goes through a momentum over- shoot after that, and shows a small period of cohesive downward motion during collapse.

[28] For all selected clouds, the pulses are relatively narrow when looking at the liquid water content and at the total water content; the onset of the pulse in terms of those variables also slightly precedes the pulse in the cloud mass graph. In Figures 4 and 5, the onset of a pulse is 

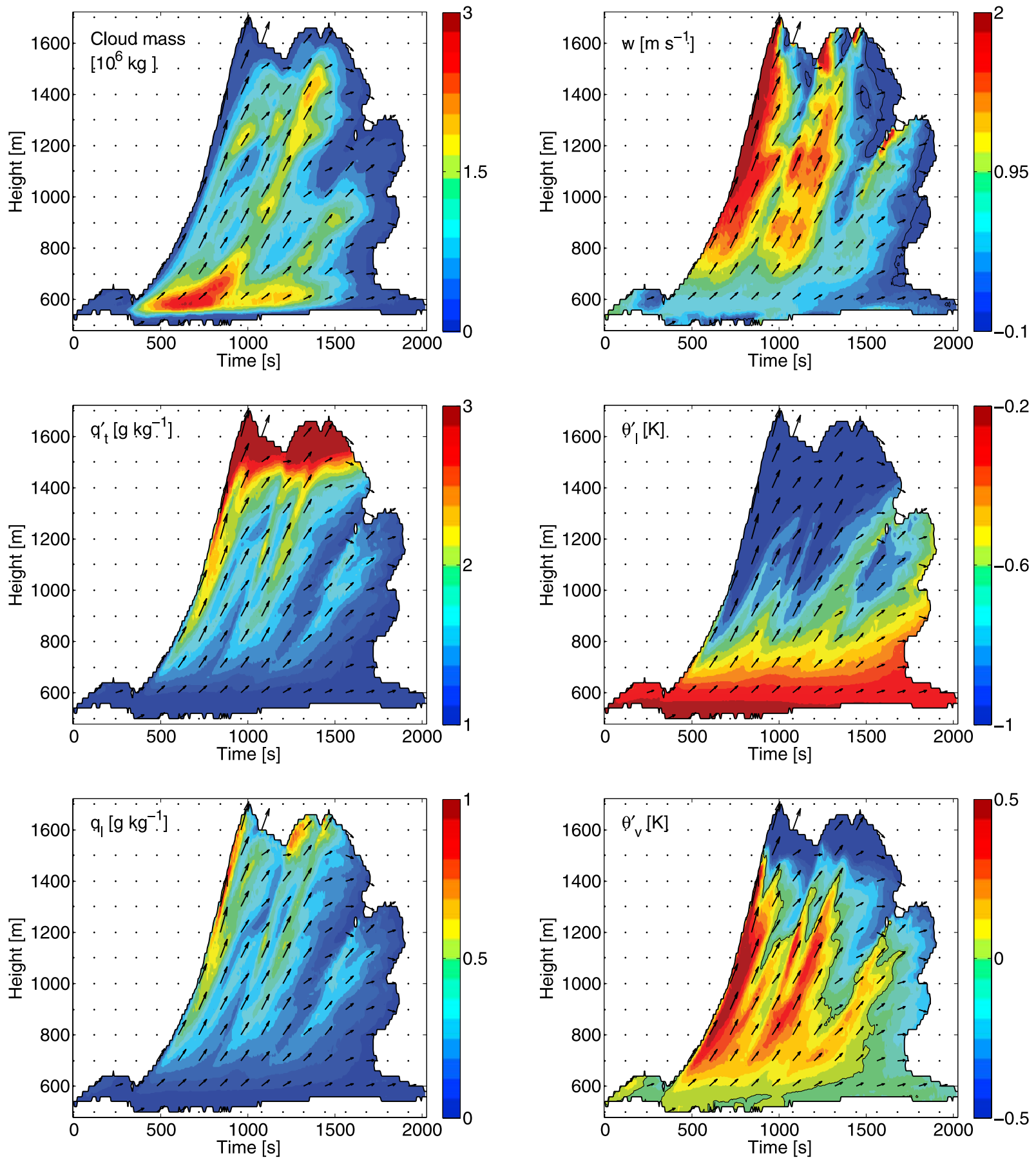

Figure 5. Height-time plots of the cloud area averaged mean properties for cloud B. The thick line denotes the cloud top and cloud base; the thin contour line denotes the 0 -isoline. The vector field denotes the cloud averaged vertical velocity. See Figure 4 for further explanation of the variables.

clearly triggered by an excess in moisture, an increase in buoyancy, followed by an increase in cloud mass and vertical velocity. The vertical velocity shows, due to the inertia of the pulse, slower adjustment to the pulse; during the decay of a pulse, especially, the average vertical velocity remains above $1.5 \mathrm{~m} \mathrm{~s}^{-1}$ while the virtual potential temperature has already decreased below $0.1 \mathrm{~K}$. At higher levels in the cloud, the buoyancy can be negative, but, due to inertia, the pulse continues to rise.
[29] For both cloud A and cloud B, the first pulse is the strongest pulse that occur in the life of the cloud; in other clouds the second or third was sometimes stronger or of similar strength. The average vertical velocity in the pulse is in agreement with the results by ZAB. However, contrary to ZAB's findings, a general trend where every pulse is weaker than its predecessor is not observed. Large pulses sometimes split in separate branches at higher levels. The influence of a pulse on subsequent pulses is somewhat unclear here; on the one hand, a previous pulse tends to 
moisturize and cool the air and make it more favorable for future pulses. On the other hand, the strong downdrafts at the end of a pulse lifetime often prevent the subsequent pulse from fully developing. The latter effect is especially strong for the pulse that reaches the cloud top first (in the case of cloud A and B: the first pulse); the overshoot this pulse goes through enhances the downdrafts. After this topreaching pulse, conditions for the subsequent pulses are more similar, and any decay in strength between the pulses should originate from a decaying subcloud thermal.

[30] Finally, it is interesting to note the remnants of the convective inhibition (CIN) slightly above cloud base. As could also be observed in the mean profiles of Figure 3, buoyancy is close to zero between cloud base and $600 \mathrm{~m}$, as is the liquid water content. In the remainder of this paper, we shall name this layer the "CIN layer" for brevity, although this does not mean that there is any CIN (i.e., negative buoyancy) left inside the cloud within this layer. Nevertheless, the in-cloud buoyancy within this layer is close to zero, and so is the vertical velocity. Furthermore, the CIN layer is relatively well mixed over its height, and in contrast with higher levels in the cloud, the pulse is not yet visible in any of the variables, again suggesting that the pulse is not driven by the subcloud layer. In the earliest life stages of the cloud, i.e., up to about $400 \mathrm{~s}$, the cloud top does not exceed $800 \mathrm{~m}$ and the cloud remains restricted to the CIN layer and behaves more or less like a forced (nonbuoyant) cloud. Similar to what happens in the transition from shallow cumulus to deep convection [Khairoutdinov and Randall, 2002; Kuang and Bretherton, 2006], these shallow cumuli themselves need to moisten their immediate environment before the CIN can be overcome.

[31] If we neglect mixing between cloud and environment, vertical advection would be the only way a parcel of air could travel through the clouds, and a strong correlation between the coherent structures and the vector field that depicts the vertical velocity can be observed. This holds reasonably well for the updrafts. The updrafts show as arrows pointed toward the top-right corner of the diagram, with a steeper slope (due to acceleration) as the updraft ascends. The slope of the vector field is similar to the slope of, for instance, the structure of the $\theta_{\mathrm{v}}^{\prime}$ field; in other words the phase speed equals to the advective velocity. However, the correlation between the phase speed and $w$ does not hold at all during the decay of the pulse, where the vertical velocity goes to zero or becomes negative, but information still propagates upward, which can be observed for instance for cloud B on the line between $(t=1300 \mathrm{~s}, z=1000 \mathrm{~m})$, and $(t=1600 \mathrm{~s}, z=1300 \mathrm{~m})$. Only the downdrafts at the cloud top (e.g., in cloud A from $(t=1100 \mathrm{~s}, z=1800 \mathrm{~m})$ to $(t=1300 \mathrm{~s}, z=1400 \mathrm{~m}))$, are able to propagate in the lowerright direction. Other downdrafts that are located just below a pulse and propagate upward, along with the pulse. This is the signature of a shedding thermal under influence of buoyancy sorting [Blyth et al., 1988]. The downdraft is constantly created in the wake of a bubble by air that is less buoyant than the top part of the bubble. This air slows down and mixes away into the environment, and evaporates. This way, the downdraft leaves the cloud and thus by definition, the downdraft also leaves the ensemble.

[32] To study the time-dependent behavior of the cloudenvironment interaction, the near-cloud region can be taken into account. This near-cloud region can be defined in several ways; ZAA defined it by the concentration of a passive tracer that originated in the subcloud layer, and Couvreux and Rio [2008] showed that isosurfaces of $q_{\mathrm{t}}^{\prime}$ are also a reliable marker of the near-cloud region. Heus and Jonker [2008], Jonker et al. [2008], and Heus et al. [2008, 2009] showed that a subsiding shell can be expected in an area of $200 \mathrm{~m}$ immediately around the cloud, and that the further environment shows much less motion. However, hardly anything is known about the behavior of the shell in time. So here we define the shell as all environmental air that resides within $200 \mathrm{~m}$ from the nearest in-cloud point at that height, and we can define the shell averaged properties of a cloud as:

$$
\bar{\phi}^{s}(z, t)=\frac{1}{A_{s}} \int_{A_{s}} \phi d x d y
$$

with $A_{s}=A_{s}(z, t)$ the area of the shell. In Figures 6 and 7, the mean excesses (relative to the domain averaged values) of liquid water potential temperature, total water content, and virtual potential temperature and the mean vertical velocity of the shell are presented for cloud A and cloud B. Again, the vector field depicts the in-cloud mean vertical velocity.

[33] The liquid water potential temperature and the total water content excesses are roughly anticorrelated. This results in a virtual potential temperature that follows the liquid water potential temperature in a subdued manner. For most of the time and most of the heights, the conserved variables $\theta_{1}$ and $q_{t}$ mix linearly and display values between their in-cloud and slab averaged environmental value. The virtual potential temperature and vertical velocity are negative, meaning that a subsiding shell is indeed present. However, patches of positive excess in the liquid water potential temperature (and negative excess in the total water content) are very pronounced. The only possible source of this air is from higher levels of the environment. Presumably, this is air dragged down by the shell that is induced at higher levels. Note, however, that the excesses are relatively small; an excess in liquid water potential temperature of $0.1 \mathrm{~K}$, or a deficit in total water content of $-0.1 \mathrm{~g} \mathrm{~kg}^{-1}$, suggests that this air has been dragged down by about $20 \mathrm{~m}$. This relatively small displacement can also be observed in the upward pointing isolines of the vertical velocity, even for regions with negative velocity in the shell. The coherent structures correlate better with the in-cloud velocity as shown in the vector field, than with the average velocity of the subsiding shell itself. Clearly, the dynamics in the shell is a slave to the cloud system and does not propagate very far on its own.

[34] Due to the many mixing effects interacting with each other within the shell, the signature of the pulses is not as pronounced in the shell as within the cloud, despite the fact that the shell dynamics are a slave to the cloud dynamics. Some signature of the pulse can be observed in the vertical velocity, where strong environmental downdrafts directly correlate with strong pulsating growth, for instance around $(t=1000 \mathrm{~s}, z=1000 \mathrm{~m})$ in cloud B. The onset of the first pulse, the ascending cloud top (ACT), leaves a particular signature on the environment of the cloud, since the ACT travels through an undisturbed environment. This results, for a brief time (less than a minute), in environmental air 

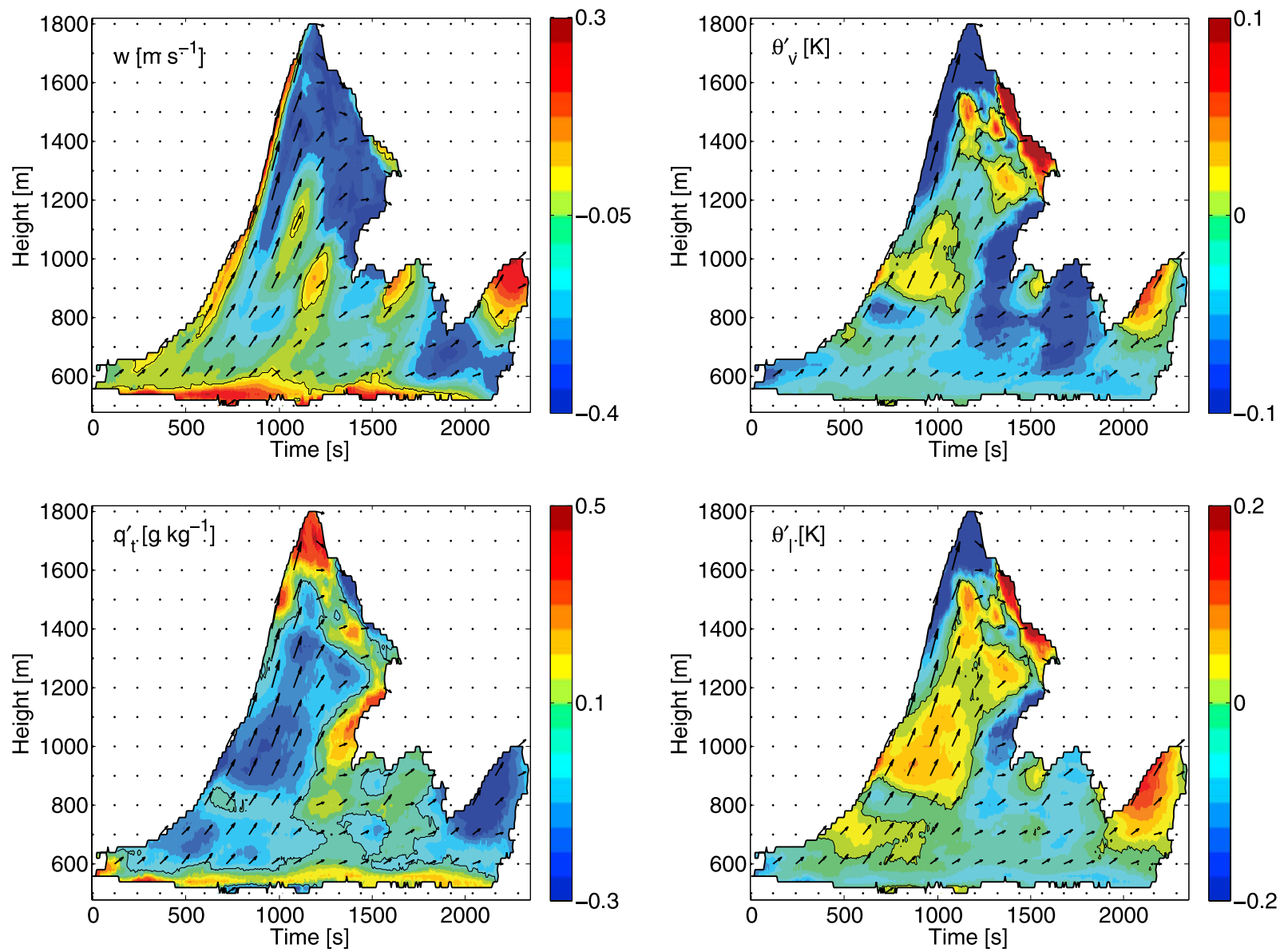

Figure 6. Height-time plots averaged over the environment within $200 \mathrm{~m}$ of cloud A. The thick line denotes the cloud top and cloud base; the thin contour line denotes the 0 -isoline. The vector field denotes the cloud averaged vertical velocity. See Figure 4 for further explanation of the variables.

being dragged upward before the effect of mixing results in downward motion in the shell. During the decay phase of the pulse, subsidence in the shell decreases, up to the point where environmental velocities can even become positive, as for instance around $(t=800 \mathrm{~s}, z=1000 \mathrm{~m})$ in cloud B. This can be explained as follows. As discussed by Heus and Jonker [2008], the vertical velocity in the shell consists of a balance between environmental air being dragged upward by the momentum of the cloud, and detrained cloudy air moving downward due to buoyancy reversal. Firstly, during the decay phase of the pulse, the in-cloud air is diluted, with values of conserved variable excesses $\phi^{\prime}$ that are around $70 \%$ of their value during the maximum strength of the pulse. If such air detrains from the cloud, only a little buoyancy reversal occurs. Secondly, the decay of the pulse correlates with a period of strong horizontal convergence, resulting in more entrainment and less detrainment. Both the decreased buoyancy reversal and decreased detrainment result in a smaller strength of the subsiding shell, and thus in a less negative, or even positive, vertical velocity in the environment.

[35] Although the pulses are still visible in the vertical velocity diagrams of Figures 6 and 7, hardly any of its signal can be found in the thermodynamic quantities. Where conservation of momentum tends to widen the region of negative vertical velocity, the region of negative buoyancy is much smaller; the buoyancy minimum always lies at or very near to the cloud edge, where the evaporative cooling is the strongest. This means that, for the thermodynamic variables, a large part of the $200 \mathrm{~m}$ wide shell consists of environmental air that is only very indirectly part of the cloud system and cannot react fast enough to changes within the cloud to accommodate for changes in the cloud properties along the life cycle of a pulse.

[36] The total mass flux through the cloud and the shell combined is equal to:

$$
\begin{aligned}
M_{T}(z, t)=M_{c}(z, t)+M_{S}(z, t)= & \int_{A_{c}} \rho w(x, y, z, t) \mathrm{d} A \\
& +\int_{A_{s}} \rho w(x, y, z, t) \mathrm{d} A,
\end{aligned}
$$

with $\rho$ the density of the air and with $A_{c}$ and $A_{s}$ the area of the cloud and the shell, respectively. They are shown for the two clouds in Figure 8.

[37] In general, these results are very similar to the ones reported by ZAA, with a positive mass flux during the first stages of the cloud lifetime and a negative mass flux afterward. Like ZAA, the negative mass flux is mainly 

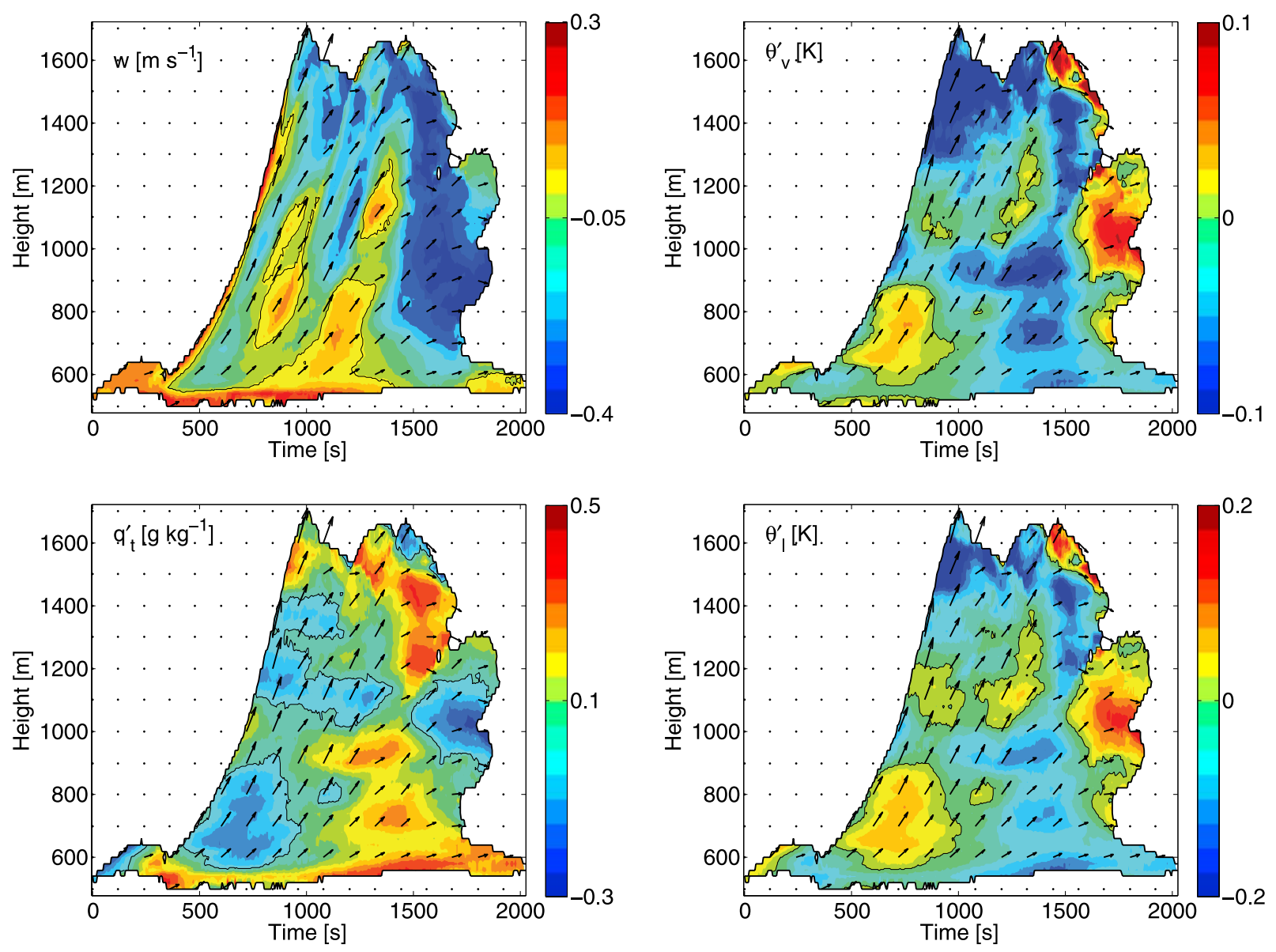

Figure 7. Height-time plots averaged over the environment within $200 \mathrm{~m}$ of cloud B. The thick line denotes the cloud top and cloud base; the thin contour line denotes the 0 -isoline. The vector field denotes the cloud averaged vertical velocity. See Figure 4 for further explanation of the variables.

caused by significant downdrafts in the shell; the mass flux inside the cloud remains positive even though $M_{T}$ has become negative. However, while ZAA argued that the regime change from positive to negative mass flux propagates downward in time for large clouds and upward in time for smaller clouds, neither can be concluded from our results. Instead, the regime change from an upward transport in the cloud system to a downward transport goes on a very short, that is, within $200 \mathrm{~s}$ over the entire $1000 \mathrm{~m}$ high cloud, without much correlation with the vertical velocity vector field. This means that, regardless of the exact cause of this transition, information about the change travels through the cloud with a speed of $5 \mathrm{~m} \mathrm{~s}^{-1}$, much higher than the maximum velocity observed in the cloud at any time or height. The reasons behind this sudden transition remains somewhat unclear thus far, especially since the
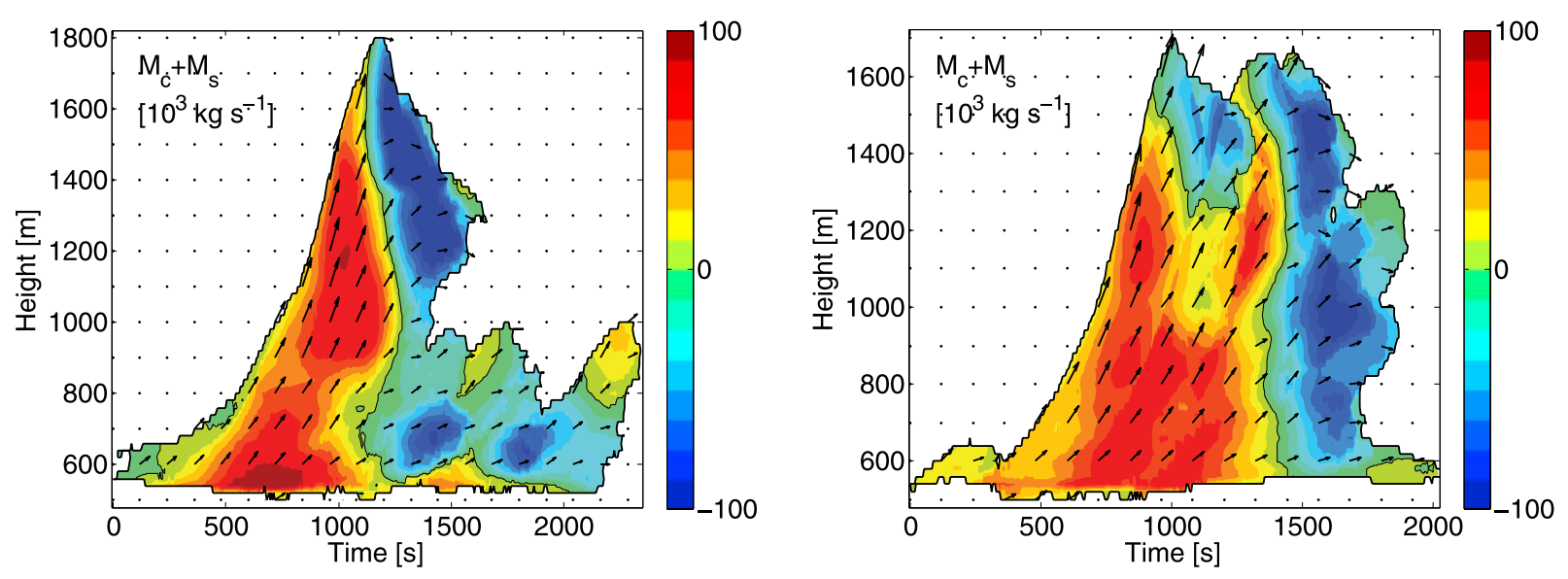

Figure 8. The mass flux integrated over the cloud and the shell for (left) cloud A and (right) cloud B. 

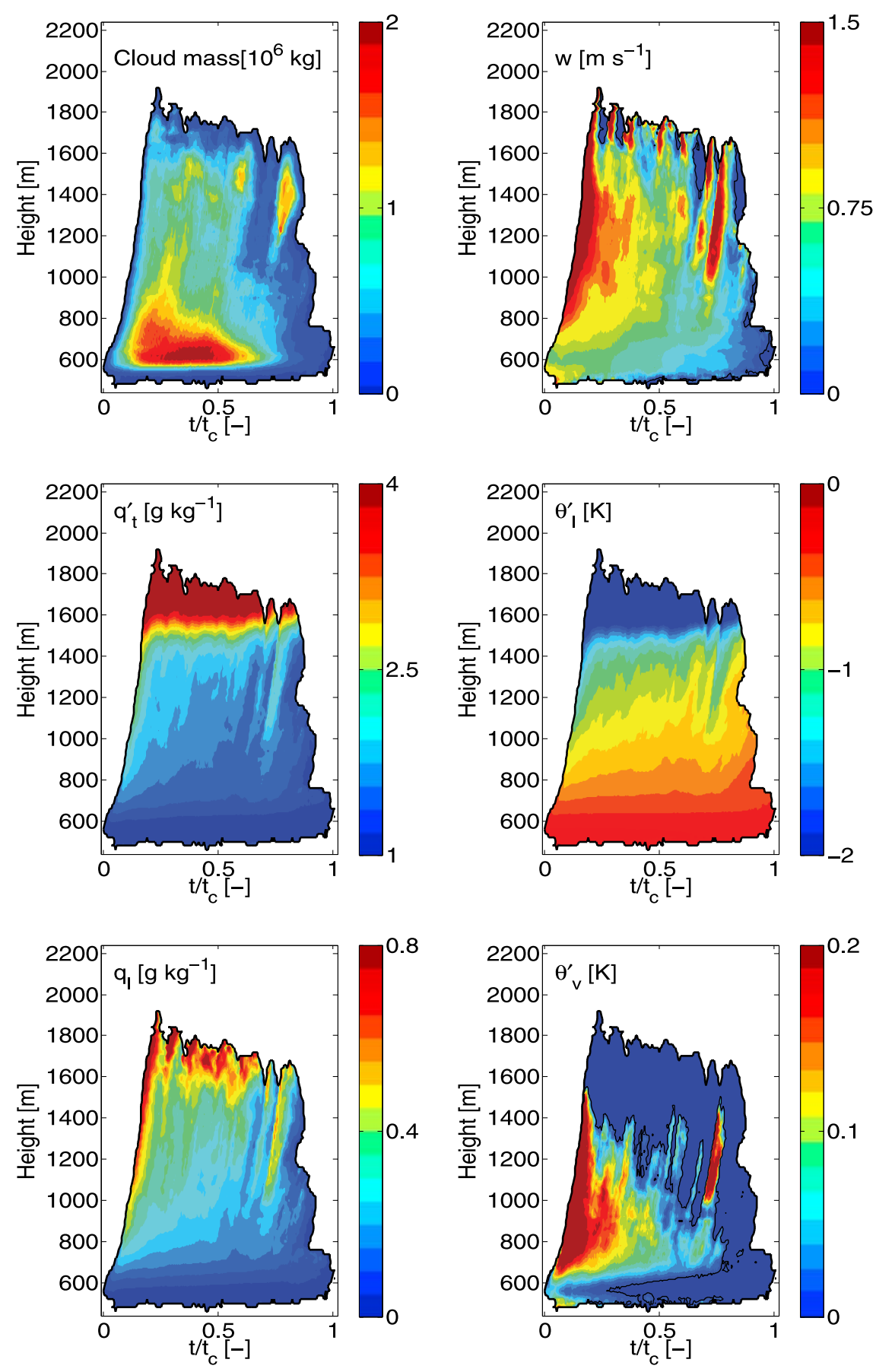

Figure 9. Thermodynamical properties averaged over the lifetime of clouds and scaled with the lifetime $t_{\mathrm{c}}$ of each cloud. See Figure 4 for further explanation of the variables.

heights that are the first to display a negative mass flux are not associated with the heights where the cloud breaks up in several pieces; the looming break up does not seem to be the cause of the transition.

\section{Statistical Representation}

[38] Of the processes discussed in section 3, only the total mass flux (Figure 8) had a clear dependency on the life cycle of the entire cloud. The other features, especially inside the cloud, are more governed by the life cycle of the pulses. In a sense, a cloud can be seen as a sequence of one or more pulses, and the lifetime is defined by the number of pulses a cloud consists of. In Figure 9, a composite cloud has been built by scaling all selected clouds with their individual lifetime $t_{\mathrm{c}}$. This way, a natural emphasis lies on the beginning and the end of the cloud life cycle, because these are automatically synchronized by this procedure. The decline in cloud mass, and the negative buoyancy, especially, at the end of the cloud life cycle are clearly visible. However, because the mature phase of the cloud is not so well synchronized, much of the pulse-like structure is 


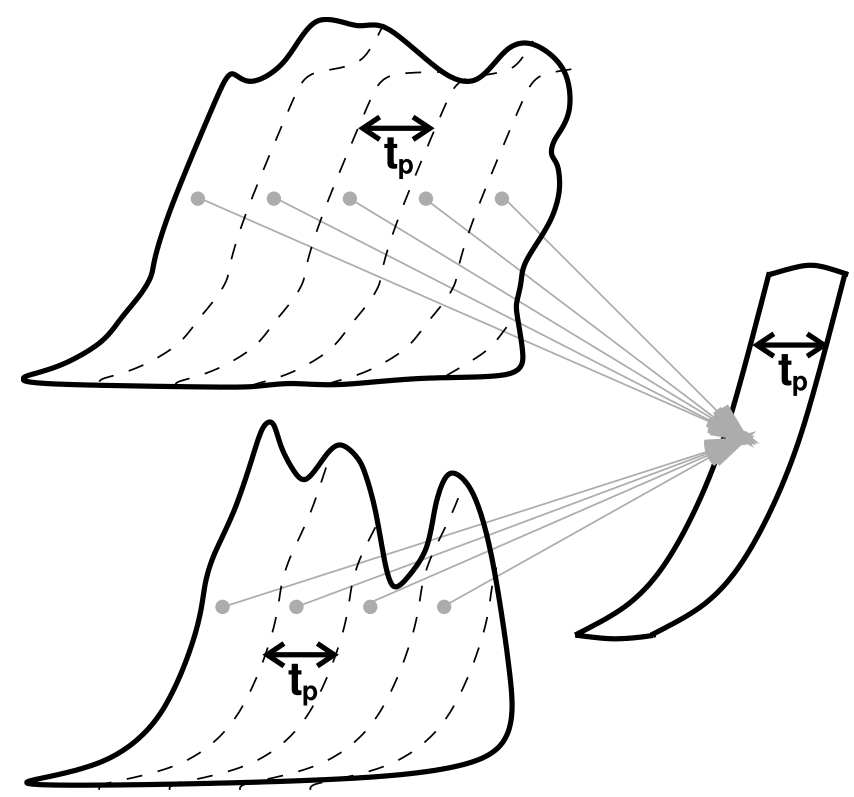

Figure 10. A schematical overview of the averaging procedure over all pulses. All clouds are divided in pulses between minima of the liquid water content at $800 \mathrm{~m}$, and quantities are conditionally sampled with their time and height in the pulse.

averaged out. Thus an important element of the cloud life cycle is ignored.

[39] Therefore if we want to do a life cycle analysis, it stands to reason to do a life cycle analysis over the pulse instead of over the entire cloud. To perform such an analysis, an averaging procedure as schematically drawn in Figure 10 is used.

[40] Each of the 35 clouds selected from the BOMEX data set is divided up in pulses, that is, in intervals between two minima of liquid water content at $z=800 \mathrm{~m}$, counting from the moment the ascending cloud top passes through the observation level. This yields an ensemble of 140 separate pulses. Similar to what Williams and Hacker [1992] did for thermals in the dry convective boundary layer, the pulses are normalized with their duration. From the 140 normalized pulses, one average pulse is composited, with the pulse onset defined as the average pass-through time of the ACT. This procedure means that the onset of the first pulse is synchronized. However, because each cloud consists on average of four pulses, much of the signal after sampling is governed by the subsequent pulses, and not by the ACT itself. Given the large number of pulses in the ensemble, coming from different life stages of different clouds, the composite pulse can be seen as a statistically reliable depiction of the entire cloud field.

[41] In Figure 11, the thermodynamic properties of the composite pulse are presented. Clearly, the fluctuations are the most intense at lower levels (below $1000 \mathrm{~m}$ ) in the buoyancy signal, and they are followed at higher altitudes (above $1000 \mathrm{~m}$ ) by an increased vertical velocity that can push the air well into the inversion region, despite the negative buoyancy.

[42] However, effects of stratification hide the appearance of the pulse in the signal of $\theta_{1}^{\prime}$ and $q_{\mathrm{t}}^{\prime}$, and, also for $\theta_{\mathrm{v}}^{\prime}$ in the inversion layer. Therefore it is interesting to look at the normalized deviations from the cloud averaged values:

$$
\phi^{\prime \prime}(z, t)=\frac{\phi(z, t)-\langle\phi\rangle(z)}{|\langle\phi\rangle(z)|},
$$

with $\phi=\left\{w, q_{1}, q_{\mathrm{t}}^{\prime}, \theta^{\prime}{ }_{w}, \theta^{\prime}\right\}$ and $\langle\phi\rangle$ is the slab averaged value, conditioned over all selected clouds; this slab average was already presented in Figure 3. By definition, this graph will always display a zero-crossing at some point in time, so it is important to look at the size and coherency of the fluctuations. These results are presented in Figure 12.

[43] In terms of liquid water content or buoyancy, the pulse flows coherently into the inversion layer (above $1500 \mathrm{~m}$ ). After reaching cloud top, the pulse breaks up in an oscillating motion. This can be interpreted as chunks of cloud that, during their dissipation phase, go through a few swift undershoots and overshoots.

[44] The fluctuations of the conserved quantities at $1200 \mathrm{~m}, q^{\prime \prime}{ }_{t}$ and $\theta^{\prime \prime} l$, are in the order of $8 \%$ of the mean in-cloud excess of these quantities. Despite these relatively small fluctuations, the effect on the buoyancy is relatively large, on the order of $30 \%$ of the mean excess. This is due to the fact that all the fluctuations in $q^{\prime \prime}{ }_{t}$ are one-to-one mirrored in the liquid water content $q^{\prime \prime}{ }_{l}$, which in turn has a strong impact on the buoyancy.

[45] Finally, in Figure 13, the differences between the first pulse of each cloud and the subsequent pulses are shown. Clearly, the cloud mass has not yet fully developed during the first pulse. The mass increases in time during the first pulse, and during the subsequent pulses the mass remains more or less constant, due to a significant amount of passive air surrounding the cloud core, that is slowly mixing with the environment. The first pulse is much stronger in terms of buoyancy than the subsequent pulses, although the general pattern remains in tact, with a more buoyant pulse top in comparison with the latter part of the pulse.

\section{Possible Mechanisms Behind Pulsating Growth}

[46] So far, we have seen that pulsating growth is important in the life cycle of cumulus clouds, but it is yet unclear what the driving mechanism is behind these pulses. Three possible mechanisms are as follows:

[47] 1. The cloud layer is decoupled from the subcloud layer, and the clouds drift from thermal to thermal. Such a process can be disregarded as unlikely, because a decoupling should result in a discontinuity in the mean horizontal velocity, which is not the case.

[48] 2. The subcloud thermal itself consists of separate pulses, for instance due to strong mean shear.

[49] 3. The pulse is part of the cloud dynamics.

[50] To investigate the possibility of the pulsating subcloud thermal, it is instructive to look at two other cases of shallow cumulus besides standard BOMEX: a modified BOMEX case, without any large-scale forcings, and the ARM case. Although the ARM case shows a mean wind of $10 \mathrm{~m} \mathrm{~s}^{-1}$, this mean wind is constant in the bulk of the subcloud layer, so a mean shear is observed only directly to the surface. In Figure 14, the buoyancy of a typical cloud from both cases is shown. In comparison with Figures 4 and 5 , both the modified BOMEX as well as the ARM case 

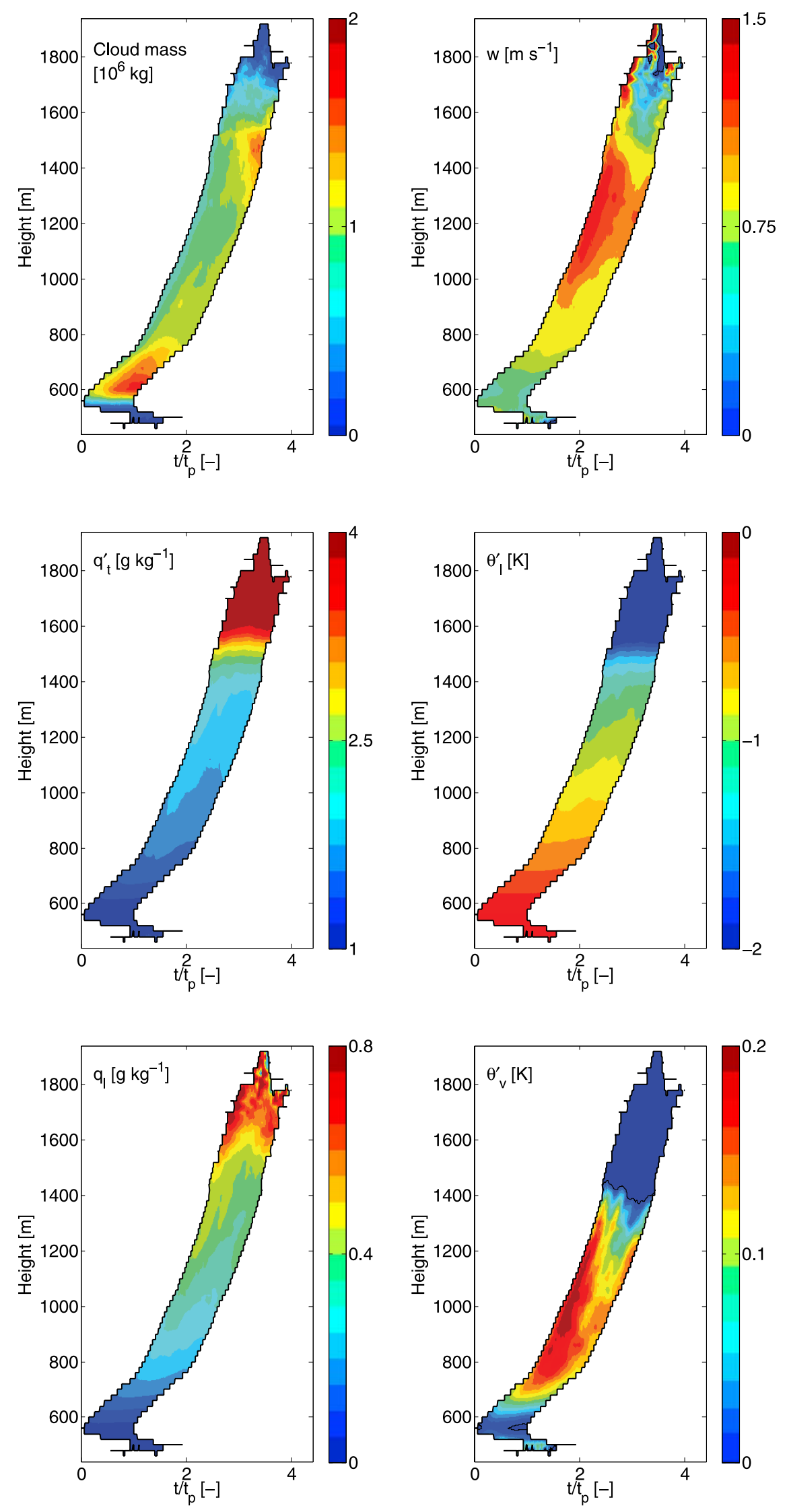

Figure 11. Thermodynamical properties of the pulse within the cloud, scaled with the average lifetime $t_{\mathrm{p}}$ of a pulse. See Figure 4 for further explanation of the variables. 

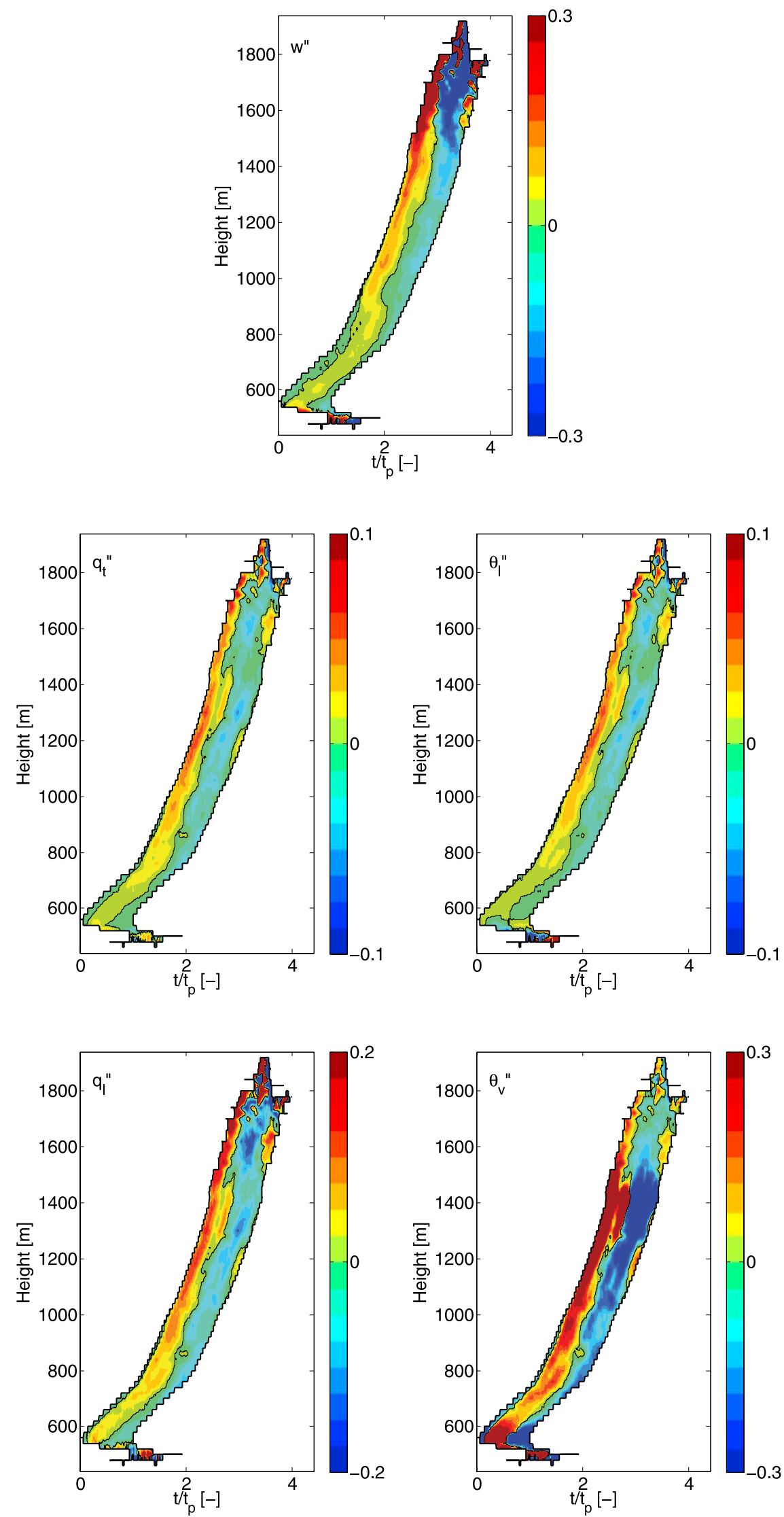

Figure 12. Fluctuations of thermodynamical properties of the pulse within the cloud. The " denotes deviations around the slab mean over all clouds. See Figure 4 for further explanation of the variables. 

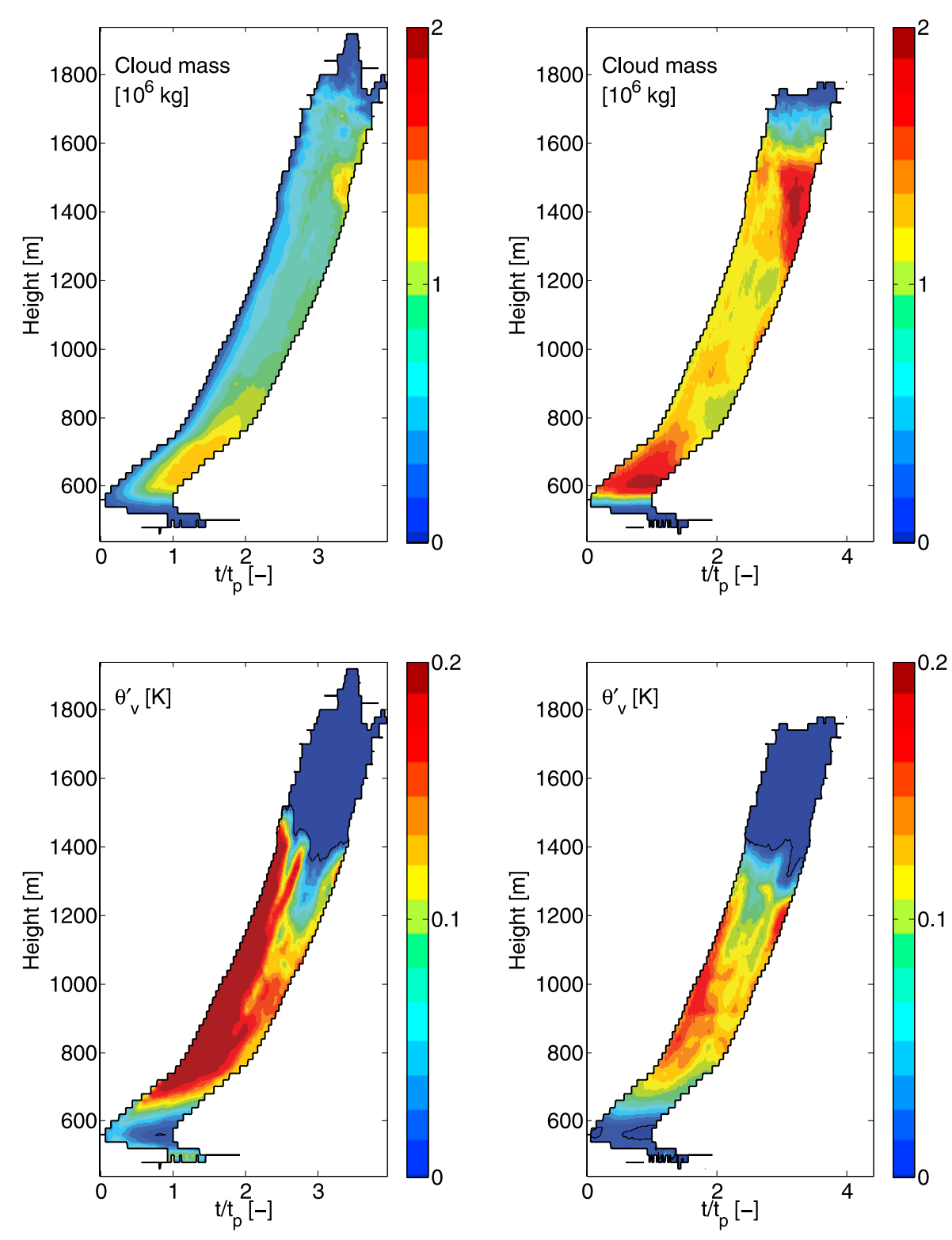

Figure 13. (top) Mass and (bottom) virtual potential temperature excess of (left) a composite first pulse of each cloud and (right) the composite over the subsequent pulses of each cloud.

show different cloud properties. For instance, the lack of large-scale subsidence results in a strongly increased cloud base height and cloud top height for the modified BOMEX case in comparison with the reference case. However, the pulsating growth remains robust, suggesting that for instance the mean shear does not influence the pulsating growth, neither by providing clear air for the next pulse to grow into, or by breaking up the subcloud thermal into bubbles. The of the pulses also remains comparable with $t_{\mathrm{p}}=408 \mathrm{~s}$ as observed in standard BOMEX.

[51] What is more, the (relatively big) CIN layer is clearly visible in the ARM case as a layer without any oscillations visible in time; this was already visible in standard BOMEX, although the CIN layer is much weaker in that case, and the effect is consequently less pronounced. Apparently, the CIN layer serves as a buffer between the subcloud thermal and the convective cloud; fluctuations in the inflow of air into the CIN layer (if any) are buffered by it and can thus not be the cause of the pulsating growth of the rest of the cloud. This is emphasized in Figure 15; for all three simulations (BOMEX, modified BOMEX, and ARM), the total water flux $w q_{\mathrm{t}}^{\prime}$ is constant at cloud base, but clearly this is not the case anymore at the top of the CIN layer. Thus we can conclude that the influence of the sub cloud layer thermal on the pulsating growth is limited.

[52] Therefore the third possible mechanism, that pulsating growth is a feature of the clouds own dynamics is the most likely candidate remaining. In Figure 16, the in-cloud mass flux $M_{c}$ and the horizontal divergence $D$ :

$$
D=\int_{A_{c}}-\frac{\partial w}{\partial z} d A
$$

are plotted. 

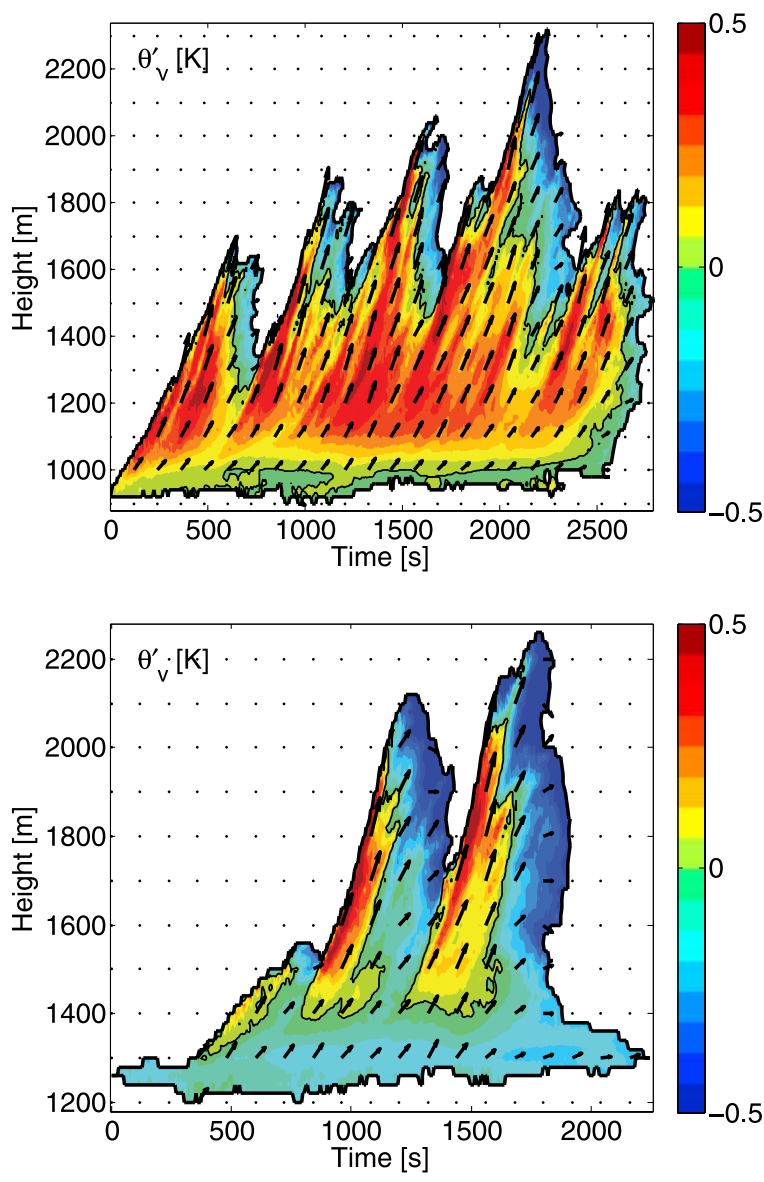

Figure 14. The mean virtual potential temperature of two clouds: (top) from BOMEX without large-scale forcings and (bottom) from ARM.

[53] Within the CIN layer (below $600 \mathrm{~m}$ ), a positive divergence due to decelerating updrafts is observed. This is in agreement with the description of forced clouds by Stull [1985]; air enters the cloud in the center, diverges to the outer part and the direct environment of the cloud, and descends to the subcloud layer again. Large outflow can be observed in the inversion region (above $1400 \mathrm{~m}$ ), and also at the ascending pulse top throughout the bulk of the cloud layer. In the region below $1400 \mathrm{~m}$ but above the CIN layer $600 \mathrm{~m}$, it is of interest to look at the precise timing of the maximum of the pulse in the virtual potential temperature $\theta^{\prime \prime}{ }_{v}$ in Figure 12, the mass flux, and the minimum in divergence. Always looking on the same height (for instance, $1000 \mathrm{~m}$ ), it can be observed that the $\theta^{\prime \prime}{ }_{v}$ maximum lies at the front of the pulse, and that this maximum precedes the maximum in mass flux. The mass flux is in its turn followed by the divergence, and the divergence has a minimum (meaning maximum convergence) near the end of the pulse, similar to mechanisms described by, for instance, Zipser and LeMone [1980], Blyth et al. [1988], and Hunt et al. [2003], in the sense that while the inflow of air may be constant, the massive entrainment yields an unstable flow pattern of puffs, or pulses. The cloud is continuously fueled with moist air from the subcloud layer. Above the CIN layer, this air becomes positively buoyant, accelerates and creates a maximum in the vertical mass flux.
This induces lateral inflow of environmental air, the pulse becomes detached from its source of supply and fades again. An important (maybe dominating) part in this mechanism could be caused by the fact that the vertical acceleration results in convergence of dry, warm air from the environment in the wake of the pulse, effectively recreating the convective inhibition. The detaching pulse due to lateral entrainment can be observed for all three cloud ensembles;
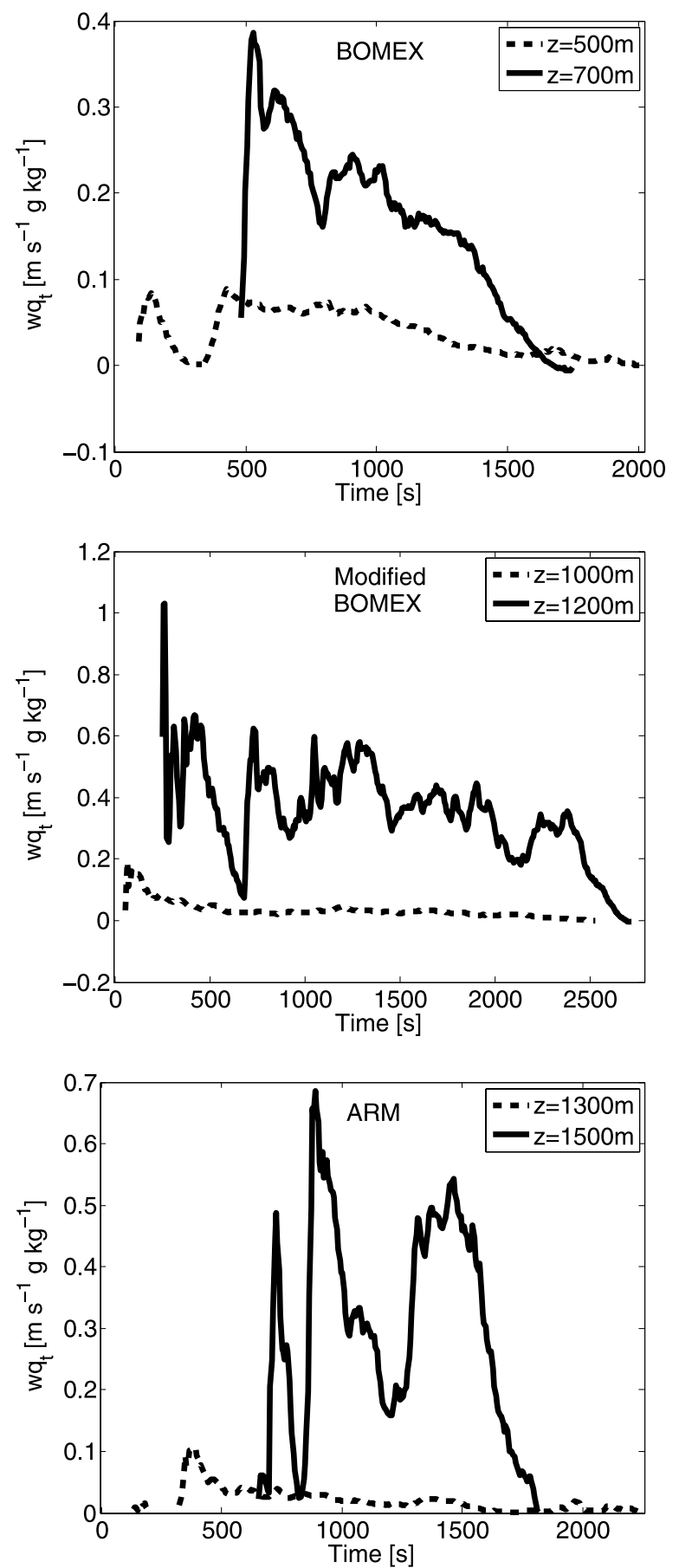

Figure 15. The in-cloud total water flux $w q_{\mathrm{t}}$ at cloud base (solid line) and at the top of the CIN layer (dashed layer): (from top to bottom) for standard BOMEX, for modified BOMEX, and for ARM. 

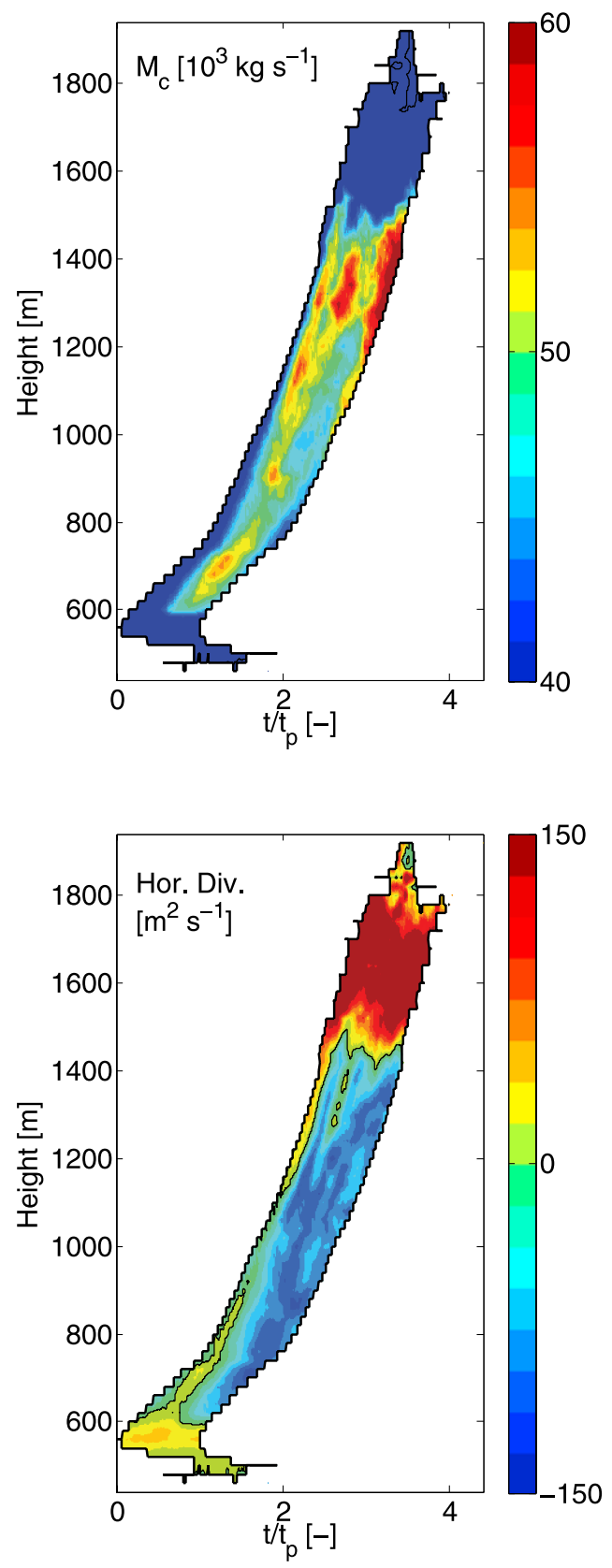

Figure 16. Mass flux and horizontal divergence in the pulse.

not only the standard BOMEX case, but also the BOMEX case without large-scale forcings and the ARM case show this mechanism to sustain pulsating growth.

\section{Conclusions}

[54] In this study, we have made an attempt to do a life cycle analysis of cumulus clouds in a statistically reliable way. The use of the VE has facilitated the selection process in such a way that a sufficient number of clouds could be selected, to the best knowledge of the authors, for the first time. Of course, using human perception to make a selection of clouds generated by numerical simulations could initiate a bias toward the clouds that are perceived as favorable by the observer. However, because conditional averages of the mean thermodynamic variables over the entire cloud field yields results similar to the values found in the averages over the selected clouds, the selection process appears to be reliable and, given the outcome, rather effective to obtain more information on the time-dependent behavior of cumulus clouds.

[55] The oscillating nature of cumulus clouds is shown to be a key factor in the understanding of the life cycle of clouds. For many purposes, a cloud can best be seen as a sequence of pulses, which is in agreement with the findings by Malkus [1952], French et al. [1999], and Zhao and Austin [2005a, 2005b].

[56] The pulses are observed in several cases, regardless of the large-scale forcings (e.g., shear) or the Bowen ratio. This means that the pulses originate from the cloud layer and, in combination with the earlier reports of the pulse, pulsating growth is clearly a generic feature of the dynamics of cumulus clouds. Since the pulses originate in the lower part of the cloud layer, but distinctly above cloud base, the lower cloud layer is the layer where the mechanism behind the pulsating growth should be found. The of the pulses matches best with the Albrecht [1981] that incorporates decay of the in-cloud excess in water content. Furthermore, strong convergence in the lower cloud layer is observed during the second phase of a pulse. All this evidence suggests that the pulsating growth is a self-property of the cloud, and that the pulses are independent from the subcloud layer.

[57] In the course of this study, the convective inhibition (CIN) layer has proven to be a small yet clearly visible layer in the cumulus cloud, and it is suggested that the CIN layer plays a crucial role in the life cycle of cumuli. First of all,

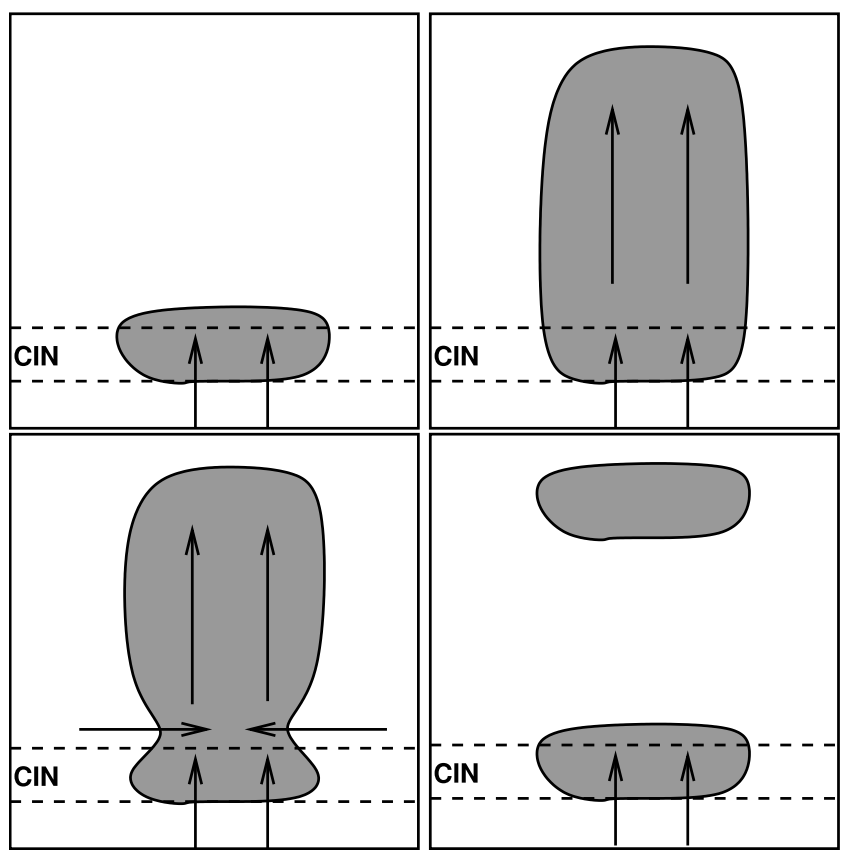

Figure 17. Conceptual picture of the pulse. A steady supply of buoyant air enters the cloud through the CIN layer, the air accelerates, generates horizontal convergence and inflow of environmental air, which causes detachment of the pulse from its source, and finally, the pulse decays. 
the CIN functions as a barrier during the initial stage that has to be overcome by a steady supply of moisture from the subcloud thermal. During this stage, the cloud complies with the classical image of a forced cloud [e.g., Stull, 1985]. The first pulse can only shoot through the cloud layer after the local CIN is sufficiently moistened and cooled. Later on in the life cycle of the cloud, the remnants of the CIN layer, that are still barely buoyant, serve as a buffer layer that releases cold, moist air from the subcloud layer with a more or less steady rate.

[58] Above the CIN layer, the pulses are driven by a system where buoyancy induces acceleration, horizontal convergence, and detachment of the pulse from its source (as sketched Figure 17). Therefore the current study could give statistical evidence for a model of pulsating growth that is similar to the models, among many others, of Zipser and LeMone [1980] for cumulonimbus clouds, to the studies of congesti by Blyth et al. [1988], or by Hunt et al. [2003] for the formation of thermals and/or plumes in the dry convective boundary layer. The fundamental difference between the dry and the cloudy convective boundary layer is that, for clouds, the instability can be much stronger due to the hostile environment around the cloud and due to the latent heat release of condensation. In terms of momentum budget, this model of pulsating growth would be a time-dependent balance between buoyancy and vertical advection. The fact that the lateral entrainment and the subsequent detachment takes place significantly above cloud base contributes to the stable foundation that keeps the cloud whole over the duration of several pulses.

[59] One feature of the cloud system (that is, the cloud and its immediate surrounds) cannot be captured by the pulse-like model of the cloud: at some point during the life cycle of the cloud system, the total mass flux of the system switches from net upward to net downward, see Figure 8. From that moment on, the upward mass flux within the cloud is dominated by the downward mass flux in the shell. In contrast with ZAA, this change of sign seems to occur within a short time over the entire height of the cloud, suggesting that information about the change has traveled through the cloud with a speed higher than the maximum velocity observed within the cloud. While this transition in itself is not necessarily very important, the high velocity of the information is something that could not be easily explained, and it might be of interest for further research.

[60] While several features of the evolution of cumulus clouds in time could be addressed in this paper, the virtual reality environment allows for improved studies of many more features. One of the most prominent topics is to investigate the role of the subcloud layer thermal on the cloud. Similar to the approach of selecting the clouds in this paper, the subcloud thermal could be selected with a combination of predefined rules (e.g., a threshold in $q_{t}$ or Lagrangian particles backtracking the flow from cloud base) and of observations to remove obvious outliers from the statistics. Based on the current work, no role of the subcloud layer on the pulsating growth is expected. However, the role of the thermal on other aspects of the cloud life cycle remains an interesting line of research.

[61] Acknowledgments. The authors would like to thank Remco Verzijlbergh and Stephan de Roode (both TUD) for the numerous dis- cussions. The investigations were supported by the Netherlands Organization for Scientific Research. This work was sponsored by the National Computing Facilities Foundation for the use of supercomputer facilities.

\section{References}

Albrecht, B. A. (1981), Parameterization of trade cumulus cloud amounts, J. Atmos. Sci., 38, 97-105, doi:10.1175/1520-0469(1981)038<0097: POTCCA $>2.0 . \mathrm{CO} ; 2$.

Berg, L. K., and R. B. Stull (2005), A simple parameterization coupling the convective daytime boundary layer and fair-weather cumuli, J. Atmos. Sci., 62(6), 1976-1988, doi:10.1175/JAS3437.1.

Blyth, A. M. (1993), Entrainment in cumulus clouds, J. Appl. Meteorol., 32, 626-641, doi:10.1175/1520-0450(1993)032<0626:EICC >2.0.CO;2.

Blyth, A. M., W. A. Cooper, and J. B. Jensen (1988), A study of the source of entrained air in Montana cumuli, J. Atmos. Sci., 45(24), 3944-3964, doi:10.1175/1520-0469(1988)045<3944:ASOTSO >2.0.CO;2.

Blyth, A. M., S. G. Lasher-Trapp, and W. A. Cooper (2005), A study of thermals in cumulus clouds, Q. J. R. Meteorol. Soc., 131(607), 11711190, doi:10.1256/qj.03.180.

Bretherton, C. S., and P. K. Smolarkiewicz (1989), Gravity waves, compensating subsidence and detrainment around cumulus clouds, J. Atmos. Sci., 46(6), 740-759, doi:10.1175/1520-0469(1989)046<0740: GWCSAD $>2.0 . \mathrm{CO} ; 2$

Brown, A. R., et al. (2002), Large-eddy simulation of the diurnal cycle of shallow cumulus convection over land, Q. J. R. Meteorol. Soc., 128(582), 1075-1093, doi:10.1256/003590002320373210.

Carpenter, R. L., K. K. Droegemeier, and A. M. Blyth (1998), Entrainment and detrainment in numerically simulated cumulus congestus clouds. Part I: General results, J. Atmos. Sci., 55(23), 3417-3432, doi:10.1175/15200469(1998)055<3417:EADINS >2.0.CO;2.

Couvreux, F., and C. Rio (2008), A conditional sampling of boundary layer plumes for validation of mass flux parameterizations, in The 18th Symposium on Boundary-Layers and Turbulence, Stockholm, Sweden, Am. Meteorol. Soc.

Cuijpers, J. W. M., and P. G. Duynkerke (1993), Impact of skewness and nonlocal effects on scalar and buoyancy fluxes in convective boundary layers, J. Atmos. Sci., 55, 151-162, doi:10.1175/1520-0469(1998) $055<0151:$ IOSANE $>2.0 . \mathrm{CO} ; 2$.

French, J. R., G. Vali, and R. D. Kelly (1999), Evolution of small cumulus clouds in Florida: Observations of pulsating growth, Atmos. Res., 52, 143-165, doi:10.1016/S0169-8095(99)00024-1.

Grabowski, W. W., and T. L. Clark (1991), Cloud-environment interface instability: Rising thermal calculations in two spatial dimensions, J. Atmos. Sci., 48(4), 527-546, doi:10.1175/1520-0469(1991)048<0527: CIIRTC $>2.0 . \mathrm{CO} ; 2$.

Grabowski, W. W., and T. L. Clark (1993a), Cloud-environment interface instability. part II: Extension to three spatial dimensions, J. Atmos. Sci., 50(4), 555-573, doi:10.1175/1520-0469(1993)050<0555:CEIIPI $>2.0$. $\mathrm{CO} ; 2$.

Grabowski, W. W., and T. L. Clark (1993b), Cloud-environment interface instability. part III: Direct influence of environmental shear, J. Atmos. Sci., 50(23), 3821-3828, doi:10.1175/1520-0469(1993)050<3821: CEIIPI $>2.0 . \mathrm{CO} ; 2$

Griffith, E. J., F. H. Post, M. Koutek, T. Heus, and H. J. J. Jonker (2005), Feature tracking in VR for cumulus cloud life-cycle studies, in Virtual Environments 2005, edited by E. Kjems and R. Blach, pp. 121-128, Eurographics.

Grinnell, S. A., C. S. Bretherton, D. E. Stevens, and A. M. Fraser (1996), Vertical mass flux calculations in Hawaiian trade cumulus clouds from dual-Doppler radar, J. Atmos. Sci., 53(13), 1870-1886, doi:10.1175/ 1520-0469(1996)053<1870:VMFCIH $>2.0 . \mathrm{CO} ; 2$.

Haiden, T. (1996), Generalization of Albrecht's cumulus cloud amount parameterization, J. Atmos. Sci., 53(21), 3164-3167, doi:10.1175/ 1520-0469(1996)053<3164:GOACCA $>2.0 . C O ; 2$

Heus, T., and H. J. J. Jonker (2008), Subsiding shells around shallow cumulus clouds, J. Atmos. Sci., 65(3), 1003-1018, doi:10.1175/ 2007JAS2322.1

Heus, T., G. van Dijk, H. J. J. Jonker, and H. E. A. van den Akker (2008), Mixing in shallow cumulus clouds studied by Lagrangian particle tracking, J. Atmos. Sci., 65(8), 2581-2597, doi:10.1175/2008JAS2572.1.

Heus, T., C. F. J. Pols, H. J. J. Jonker, H. E. A. Van den Akker, and D. H. Lenschow (2009), Observational validation of the compensating mass flux through the shell around cumulus clouds, Q. J. R. Meteorol. Soc., 135, 101-112, doi:10.1256/qj.08.66.

Heymsfield, A. J., P. N. Johnson, and J. E. Dye (1978), Observations of moist adiabatic ascent in northeast Colorado cumulus congestus clouds, J. Atmos. Sci., 35(9), 1689-1703, doi:10.1175/1520-0469(1978)035< 1689:OOMAAI $>2.0 . \mathrm{CO} ; 2$

Hunt, J. C. R., A. J. Vrieling, F. T. M. Nieuwstadt, and H. J. S. Fernando (2003), The influence of the thermal diffusivity of the lower boundary on 
eddy motion in convection, J. Fluid Mech., 491, 183-205, doi:10.1017/ S0022112003005482.

Jonker, H. J. J., T. Heus, and P. P. Sullivan (2008), A refined view of vertical transport by cumulus convection, Geophys. Res. Lett., 35, L07810, doi:10.1029/2007GL032606.

Khairoutdinov, M. F., and D. A. Randall (2002), Similarity of deep continental cumulus convection as revealed by a three-dimensional cloud-resolving model, J. Atmos. Sci., 59(17), 2550-2566, doi:10.1175/ 1520-0469(2002)059<2550:SODCCC >2.0.CO;2.

Kuang, Z., and C. S. Bretherton (2006), A mass-flux scheme view of a high-resolution simulation of a transition from shallow to deep cumulus convection, J. Atmos. Sci., 63, 1895-1909, doi:10.1175/JAS3723.1.

Malkus, J. S. (1952), The slopes of cumulus clouds in relation to external wind shear, Q. J. R. Meteorol. Soc., 78(338), 530-542, doi:10.1002/ qj.49707833804.

Perry, K. D., and P. V. Hobbs (1996), Influences of isolated cumulus clouds on the humidity of their surroundings, J. Atmos. Sci., 53(1), 159-174, doi:10.1175/1520-0469(1996)053<0159:IOICCO $>2.0 . \mathrm{CO} ; 2$.

Rauber, R. M., et al. (2007), Rain in (shallow) cumulus over the ocean The RICO campaign, Bull. Am. Meteorol. Soc., 88(12), 1912-1928, doi:10.1175/BAMS-88-12-1912.

Scorer, R. S., and F. H. Ludlam (1953), Bubble theory of penetrative convection, Q. J. R. Meteorol. Soc., 79(339), 94-103, doi:10.1002/ qj.49707933908.

Siebesma, A. P., et al. (2003), A large eddy simulation intercomparison study of shallow cumulus convection, J. Atmos. Sci., 60(10), $1201-$ 1219, doi:10.1175/1520-0469(2003)60<1201:ALESIS >2.0.CO;2.

Stull, R. (1985), A fair-weather cumulus cloud classification scheme for mixed-layer studies, J. Clim. Appl. Meteorol., 24(1), 49-56, doi:10.1175/ 1520-0450(1985)024<0049:AFWCCC $>2.0 . C O ; 2$.
Stull, R. B. (1988), An Introduction to Boundary Layer Meteorology, Kluwer Acad., Dordrecht, Netherlands.

Taylor, R. M., II, T. C. Hudson, A. Seeger, H. Weber, J. Juliano, and A. T. Helser (2001), VRPN: A device-independent, network-transparent VR peripheral system, in Proceedings of the ACM Symposium on Virtual Reality Software and Technology 2001, pp. 55-61, ACM, New York.

Williams, A. G., and J. M. Hacker (1992), The composite shape and structure of coherent eddies in the convective boundary layer, Bound. Layer Meteorol., 61(3), 213-245, doi:10.1007/BF02042933.

Zhao, M., and P. H. Austin (2005a), Life cycle of numerically simulated shallow cumulus clouds. part I: Transport, J. Atmos. Sci., 62, 1269-1290, doi:10.1175/JAS3414.1.

Zhao, M., and P. H. Austin (2005b), Life cycle of numerically simulated shallow cumulus clouds. part II: Mixing dynamics, J. Atmos. Sci., 62, 1291-1310, doi:10.1175/JAS3415.1.

Zhu, P., and W. Zhao (2008), Parameterization of continental boundary layer clouds, J. Geophys. Res., 113, D10201, doi:10.1029/2007JD009315.

Zipser, E. J., and M. A. LeMone (1980), Cumulonimbus vertical velocity events in GATE. part II: Synthesis and model core structure, J. Atmos. Sci., 37(11), 2458-2469, doi:10.1175/1520-0469(1980)037<2458: CVVEIG $>2.0 . \mathrm{CO} ; 2$.

E. J. G. Griffith and F. H. Post, Department of Mediamatics, Delft University of Technology, P.O. Box 5046, NL-2600 GA Delft, Netherlands.

T. Heus and M. Koutek, Royal Netherlands Meteorological Institute, Wilhelminalaan 10, 3732 GK, De Bilt, Netherlands.

H. J. J. Jonker and H. E. A. Van den Akker, Department of Multi-Scale Physics, Delft University of Technology, P.O. Box 5046, NL-2600 GA Delft, Netherlands. (h.j.j.jonker@tudelft.nl) 